\title{
Lipid binding properties of human ApoD and Lazarillo-related Lipocalins: functional implications for cell differentiation.
}

\author{
Mario RUIZ ${ }^{1}$, Diego SANCHEZ ${ }^{1 *}$, Colin CORRENTI ${ }^{2}$, Roland K. STRONG ${ }^{2}$ and \\ Maria D. GANFORNINA ${ }^{1 *}$ \\ ${ }^{1}$ Instituto de Biología y Genética Molecular-Departamento de Bioquímica y Biología \\ Molecular y Fisiología, Universidad de Valladolid-CSIC, 47003, Valladolid, Spain. \\ ${ }^{2}$ Division of Basic Sciences, Fred Hutchinson Cancer Research Center, Seattle, WA \\ 98109, USA \\ * MDG and DS contributed equally to this work.
}

Running title: Lazarillo-ApoD binding properties

Abstract word count: 248

Introduction word count: 632

Materials and Methods word count: 914

Results and Discussion word count: 3234

Bibliography: 81 references

Legends word count: 611

Number of figures: 7

Number of tables: 1

Number of Supplementary figures: 6

Keywords: ligand binding, fluorescence titration, neuronal differentiation; neurite extension, NLaz.

\author{
$\S$ Author for correspondence: \\ M.D. Ganfornina. \\ Instituto de Biología y Genética Molecular. \\ c/ Sanz y Forés 3. \\ Universidad de Valladolid-CSIC. \\ 47003 Valladolid, Spain. \\ Phone: +34-983-184814 \\ Fax: +34-983-184800 \\ e-mail: opabinia@ibgm.uva.es
}

\section{Contact information for all other authors:}

Mario Ruiz ruizgm@ibgm.uva.es

Diego Sanchez lazarill@ibgm.uva.es

Colin Correnti ccorrent@fhcrc.org

Roland K. Strong rstrong@,fhcrc.org 


\section{Abbreviations:}

2-AG, 2-Arachidonoylglycerol; 7-T, 7-Tricosene; 7,11-HD, 7,11-Heptacosadiene, 20 HD, 20-Hydroxyecdysone; $\alpha 1$-mg, $\alpha 1$-microglobulin; AA, Arachidonic Acid; ABCA1, ATP-binding cassette transport A1; AEA, Anandamide; ApoD, Apolipoprotein D;

ApoM, Apolipoprotein M; ArA, Arachidic Acid; cVA, 11-cis-vaccenyl acetate; EC, Endocannabinoids; HDL, High Density Lipoprotein; JNK, c-Jun N-terminal Kinase; $K_{D}$, apparent equilibrium dissociation constant; LA, Linoleic Acid; Laz, Lazarillo; L-PGDS, Lipocalin type Prostaglandin D Synthase; NLaz, Neural Lazarillo; NPC, Niemann-Pick type C disease; PA, Palmitic Acid; PLPE, 1-Palmitoleoyl-2-LinolenoylPhosphoethanolamine; RA, Retinoic Acid; RBP, Retinol Binding Protein; S1P, Sphingosine-1-phosphate; SM, Sphingomyelin; VLDL, Very Low Density Lipoprotein. 


\begin{abstract}
Lipocalins are a family of proteins characterized by a conserved eight-stranded $\beta$-barrel structure with a ligand binding pocket. They perform a wide range of biological functions and this functional multiplicity must relate to the lipid partner involved. Apolipoprotein D (ApoD) and its insect homologues, Lazarillo (Laz) and Neural Lazarillo (NLaz), share common ancestral functions like longevity, stress resistance and lipid metabolism regulation, coexisting with very specialized functions, like courtship behavior. Using tryptophan fluorescence titration we screened binding of fifteen potential lipid partners for NLaz, ApoD and Laz and uncovered several novel ligands with apparent dissociation constants in the low micromolar range. Retinoic acid (RA), retinol, fatty acids and sphingomyelin are shared ligands. Sterols however showed a species specific binding pattern: Cholesterol did not show strong binding to human ApoD, while NLaz and Laz did bind ergosterol. Among the Lipocalin-specific ligands, we found that ApoD selectively binds the endocannabinoid anandamide but not 2acylglycerol, and that NLaz binds the pheromone 7-tricosene but not 7,11heptacosadiene or 11-cis-vaccenyl acetate. To test the functional relevance of Lipocalin ligand-binding at the cellular level, we analyzed the effect of ApoD, Laz and NLaz preloaded with RA on neuronal differentiation. Our results show that ApoD is necessary and sufficient to allow for RA differentiating activity. Both human ApoD and Drosophila NLaz successfully deliver RA to immature neurons, driving neurite outgrowth. We conclude that ApoD, NLaz and Laz bind selectively to a different but overlapping set of lipid ligands. This multispecificity can explain their varied physiological functions.
\end{abstract}




\section{Introduction}

Lipocalins are an ancient and functionally diverse family of proteins characterized by their ability to bind and transport a variety of ligands, including in some cases bioactive lipids. Noted examples of functional lipocalin-lipid associations include (i) Retinol Binding Protein (RBP) and retinol [1], (ii) Apolipoprotein M (ApoM) and sphingosine-1-phosphate (S1P) [2] and (iii) the enzyme Prostaglandin D Synthase (LPGDS) and its substrate prostaglandin- $\mathrm{H}_{2}\left(\mathrm{PGDH}_{2}\right)$ [3]. Despite limited sequence identity, the Lipocalin three-dimensional structure: an eight-stranded antiparallel $\beta$ barrel with accessory $\alpha$ - and $3_{10}$ helices, is well conserved through evolution. The Lipocalin fold forms a cup, or calyx, with a central cavity, which serves as a ligandbinding site [4].

Apolipoprotein D (ApoD) is an extracellular glycosylated Lipocalin expressed with a wide temporal and tissue distribution. Although detected in liver, spleen, skin and placenta, ApoD is mostly present in serum and nervous system [reviewed by 5]. Plasma ApoD is mainly associated with high-density lipoproteins (HDL), but can also be found in very low- and low-density lipoproteins (VLDL, LDL) [6, 7]. ApoD nervous system expression has been studied during development [8-10], and it is known to increase during prefrontal cortex maturation, a period marked by active lipid metabolism [11]. On the other hand, ApoD is the most consistently up-regulated gene during brain aging in mammals $[12,13]$. ApoD levels are also elevated in many neurodegenerative diseases, including Alzheimer's [14], Parkinson's [15], schizophrenia [16] and multiple sclerosis [17]. In the mouse model of Niemann-Pick type C (NPC) disease [18], caused by deficiencies in cholesterol metabolism and management, ApoD expression is also up-regulated.

ApoD is considered the most ancient vertebrate Lipocalin and has homologues in many other phyla [19, 20]. Neural Lazarillo (NLaz) from the fruitfly Drosophila melanogaster and Lazarillo (Laz) from the grasshopper Schistocerca americana are two of the most studied ApoD homologues. Many ApoD, NLaz and Laz functions are conserved during evolution, and include protection against oxidative stress, and longevity and metabolism regulation [21-27]. Oxidative stress protection has been established for ApoD homologues in vivo, and the ApoD molecule itself exhibits antioxidant properties in vitro $[28,29]$. The regulation of ApoD and NLaz expression are also conserved, as both are controlled by the JNK signaling pathway under stress conditions $[22,26]$. Furthermore, ApoD and NLaz have been proposed as functional links between the nervous system and adipose tissue in both mammals and insects [22, 30].

The biochemical mechanism by which ApoD and related Lipocalins perform their function is not understood, though their hydrophobic pockets and potential ligands are obvious candidates to be explored. The wide range of tissue expression and physiological roles of ApoD, NLaz and Laz suggest that particular functions are dependent on specific ligands. Thus, determining the ligand specificity of these Lipocalins is essential to define their molecular mechanism.

Several ligands have been described for ApoD, but not without controversy. Progesterone-ApoD binding has been documented [31-35] and the complex cocrystallized [36]. In addition, arachidonic acid (AA) [34, 35], retinol, and retinoic acid (RA) [37] are in vitro ApoD ligands. However, a consensus has not been reached on whether bilirubin [7,35], odorants like E-3-methyl-2-hexenoic acid [35, 38] or other 
steroids, such as cholesterol [34, 39] and pregnenolone [33, 35], are bona fide functional ApoD ligands.

Laz shows positive binding for RA, AA, linoleic acid (LA) and palmitic acid (PA) [40]. However, the Drosophila NLaz ligand preferences have not been characterized.

The aim of this work is to identify new putative lipophilic ligands for the ApoDrelated Lipocalins, which should contribute to understand how they perform their physiological functions in different tissues and cellular contexts. Placing these results in an evolutionary context will help us distinguish derived versus ancestral protein-lipid functional associations. Finally, we assay at the cellular level the effect of ApoD-RA binding pair on a particular biological function: the differentiation of neurons in culture.

\section{Results and Discussion}

\subsection{Binding properties of ApoD and its related insect homologues.}

Intrinsic fluorescence titration was used to characterize the ligand binding capacities of ApoD, NLaz and Laz, an approach that has been successfully employed in many other Lipocalin binding studies [e.g.: 37, 40]. A panel of 15 small hydrophobic molecules was tested, including lipids with different size, shape, chemical properties and biological functions. Ligands identified in other studies were also included.

Previous studies were carried out using proteins recombinantly expressed in E.coli $[35,40]$. Since the three Lipocalins are glycoproteins, we performed our study with their glycosylated forms, avoiding undesired effects on the calyx structure or binding properties due to the lack of glycosylation. To perform the binding assays we purified the proteins either from native sources (ApoD) or from a eukaryotic expression system (NLaz and Laz).

\subsubsection{Retinol and retinoic acid: classical Lipocalin ligands.}

Retinol and retinoic acid (RA) are common ligands for most Lipocalins, including ApoD and Laz [37, 40]. The Lipocalin retinol binding protein (RBP) delivers retinol to several target tissues [41], and the physiological relevance of RBP-retinol interaction has been reported, for RBP mutations preventing retinol binding cause night blindness [42].

RA and especially retinol bind to ApoD with high affinity [37]. Our binding studies confirm that ApoD tightly binds retinol and RA (Fig. 1A and 1D) with $K_{D}$ values of 0.2 $\mu \mathrm{M}$ for the ApoD-retinol and $4 \mu \mathrm{M}$ for ApoD-RA interactions (Table 1). The higher affinity of ApoD for retinol over RA reported here is in agreement with previous results [37].

A study of bacterially-expressed recombinant grasshopper Laz binding to RA reported a $K_{D}$ in the low micromolar range [40]. Here we have confirmed the Laz-RA interaction with bacterially-expressed Laz (Fig. S3A), as well as with the glycosylated eukaryotic Laz produced by S2 cells (Fig. 1C). Binding to both forms results in similar $K_{D}$ (Table 1). Additionally, retinol was evaluated as a Laz ligand with positive results (Fig. 1F). RA and retinol have well known critical roles during vertebrate development [43]. The recent quantification of RA in locust embryos [44], previously thought to be absent in insects, suggests an involvement of Laz-retinol or -RA interactions in the Laz known functions during insect development [45]. 
Binding specificities for retinol and RA were also conserved in NLaz (Fig. 1B and $1 \mathrm{E}$ ), with $K_{D}$ values similar to those for ApoD or Laz (Table 1). Taken together, the binding of retinol or RA to both NLaz and Laz suggests a putative conserved physiological role for ApoD-related Lipocalins in insects, which lack a recognizable RBP ortholog [19].

To determine whether ApoD, NLaz or Laz binding to RA was dependent on the protein tertiary structure, denatured forms of all three Lipocalins were used in parallel binding assays, with the result that $\mathrm{GuHCl}$ denaturation hinders RA binding to ApoD, NLaz and Laz (Fig. S2). We conclude that an intact Lipocalin calyx is required for specific binding.

\subsubsection{Lipids present in HDL particles and plasma membrane are putative ligands for ApoD-related Lipocalins.}

ApoD was initially discovered as a component of HDL [46], where it is disulfidelinked to ApoA-II [47]. This discovery suggested that cholesterol is a potential ApoD ligand. Here we find no ApoD-cholesterol interaction in the micromolar range (Fig. 2A) in agreement with previous results where no interaction [34] or interaction in the millimolar range [39] were found, far from the low micromolar to nanomolar $K_{D}$ values typical of physiologically relevant Lipocalin-ligand interactions [48]. Although ApoD is not able to bind non-esterified cholesterol with high affinity, many studies have shown a relationship between ApoD expression and cholesterol levels in tissues or organisms, or have proposed cholesterol binding as a physiological mechanism [e.g.: 49, 50]. For example, ApoD is up-regulated in a mouse model of NPC disease [18] where cholesterol metabolism is impaired. Also, the addition of ApoD to organotypic hippocampal cultures reduced the amount of 7-ketocholesterol (an oxidized from of cholesterol) after kainate-induced excitotoxicity [51]. Although these results are suggestive of cholesterol binding, the observed correlations between ApoD and cholesterol levels cannot be ascribed to a direct cholesterol-ApoD interaction.

Drosophila cannot synthesize sterols and requires a dietary source. Ergosterol is the major sterol in the yeast-based Drosophila food used in laboratories [52]. Interestingly, NLaz and Laz can bind ergosterol with $K_{D}$ values of 2 and $2.1 \mu \mathrm{M}$ respectively (Fig. 2B-C). This finding differs from that obtained for hApoD-cholesterol interaction, and invites to test whether the lipocalin calyces and sterol binding properties are a speciesspecific divergent trait. Although the interaction might not be physiologically relevant, we tested ergosterol binding to ApoD, and cholesterol binding to NLaz and Laz. ApoD does not bind ergosterol (Fig. S4-A). Cholesterol binding is observed for NLaz, but not for Laz (Fig. S4-B and C). These results reveal structural dissimilarities among the three ligand binding pockets (see Fig. 7) and shows that ApoD binding cavity does not have properties compatible with binding to these sterols in the concentration range studied.

Sphingomyelin (SM) is the major sphingolipid in HDL particles and plasma membranes. SM has high affinity for cholesterol, and both lipids pack tightly forming lipid rafts and caveolae [53]. SM also regulates cholesterol efflux from cellular membranes to HDL via the ATP-binding cassette transporter A1 (ABCA1) [54]. A connection of ApoD with SM is supported by the enrichment in SM of ApoDcontaining blood lipoparticles [6], and by the role played by ApoD in maintaining peripheral nerve myelin sheath [55], a cell compartment that contains a high concentration of SM. Here we find that ApoD and the related insect Lipocalins NLaz and Laz bind palmitoyl-SM (Fig. 2D-F) with $K_{D}$ values of $1.3 \mu \mathrm{M}$ (ApoD), $4.6 \mu \mathrm{M}$ 
(NLaz) and $2.7 \mu \mathrm{M}$ (Laz). The Laz-SM interaction has a similar $K_{D}$ value when using the bacterially-expressed form of Laz (Fig. S3B). Thus, SM is a common ligand for this group of Lipocalins, with ApoD exhibiting a higher affinity for SM than NLaz or Laz (Table 1). The number of carbons in the sphingoid base differ across species [53] and could potentially explain the differential affinities observed. Here, we used SM with a C18:1 sphingoid base, one of the typical forms found in humans, whereas C14:0 and C14:1 are the major sphingoid base isoforms in insects [53].

Since this is the first report of binding of SM to any Lipocalin, and SM binding is conserved for ApoD, NLaz and Laz, SM could be a ligand for other Lipocalins. We tested SM binding to $\alpha 1$-microglobulin ( $\alpha 1-\mathrm{mg})$, a Lipocalin more distantly related to the ApoD group [19]. No binding interaction was detected for $\alpha 1-\mathrm{mg}$ and SM (Fig. S3D), demonstrating the specificity of this assay and suggesting that SM could be a specific ligand restricted to the ancient metazoan Lipocalins, including ApoD and its insect related Lipocalins (NLaz and Laz). Retinol binding to $\alpha 1-\mathrm{mg}$ was used as a positive control (Fig. S3C). With these findings, the question of whether ApoDcholesterol relationships found in vivo $[18,51]$ are indirectly mediated by ApoD control of SM content of membranes or lipoprotein particles is also open for further research.

Membrane phospholipids have been also suggested as ApoD ligands, though previous studies failed to show binding to phosphocholine [34]. We tested the binding of ApoD, NLaz and Laz to phosphoethanolamine, with PA as substituent in position 1 and LA in position 2 (PLPE). Neither ApoD nor its insect homologues (NLaz and Laz) showed positive binding (Fig. S4D-F). Thus, with the available data, we can discard phospholipids as probable ligands for the ApoD Lipocalin group. An interesting antecedent is found in the Lipocalin literature: ApoM is only able to bind phospholipids with high affinity when they have been previously oxidized [56]. This property is still open for ApoD and other Lipocalins and might be related to the protective functions of many family members.

\subsubsection{Binding to steroid hormones.}

ApoD was characterized as a progesterone-binding protein from the cystic fluid of women with breast gross cystic disease [31]. This was confirmed by co-crystallizing ApoD and progesterone [36], and many groups have studied the role of ApoD in breast cancer [reviewed by 57]. Steroid hormone regulatory elements have been identified in the ApoD promoter, also suggesting such a link [58]. However, we show here that ApoD does not bind 17 $\beta$-estradiol (Fig. 3A), the other main steroid hormone involved in reproduction, as previously reported [32].

The steroid hormone 20 -Hydroxyecdysone (20-E) plays crucial roles in larval molting and metamorphosis [reviewed by 59]. No binding was detected for NLaz with 20 -E in the micromolar range of concentrations (Fig. 3B), as we reported for bacterially-expressed recombinant Laz [40].

Therefore, ApoD, NLaz and Laz show a varied specificity for steroids. ApoD binds progesterone with high affinity, but it does not bind cholesterol, ergosterol or 17 $\beta$ estradiol. A possible explanation could be the presence of a hydroxyl substituent in position 3 of the cholesterol, ergosterol and $17 \beta$-estradiol molecules, which is replaced with a carboxyl group in progesterone. On the other hand, NLaz and Laz bind ergosterol but not 20-HE, which has two hydroxyl groups in positions 2 and 3. Binding of NLaz to 
both ergosterol and cholesterol implies intermediate structural and surface properties in the NLaz Lipocalin calyx.

\subsubsection{Fatty acids and related lipids.}

Fatty acids have been frequently identified as Lipocalin ligands [reviewed by 48]. Both ApoD and Laz bind AA (20:4) [34, 35, 40], which is released from membrane phospholipids by phospholipase-A2, is a substrate for eicosanoids synthesis, and plays a critical role in inflammation and tissue homeostasis. Protection of AA oxidation by binding to ApoD, preventing its conversion to isoprostanes, has been suggested as a putative ApoD mechanism of action [51].

The relationship between ApoD and AA is also extended to neurological disorders. ApoD levels are increased in certain brain areas of patients with Alzheimer's disease [14], Parkinson's disease [15] or schizophrenia [16]. A common characteristic in these three pathologies is a reduction in the levels of AA in the brain [60-62]. Increased ApoD levels could be interpreted as an attempt to correct for low AA levels, to maintain the fluidity properties of plasma membranes and the function of AA signaling pathways.

As ApoD-AA binding has already been well established, we tested if NLaz can bind other fatty acids such as arachidic acid (ArA) or PA, which are abundant in Drosophila sphingolipids and glycerophospholipids respectively [52]. Figure 4B-C shows a positive binding with ArA and PA. The estimated $K_{D}$ were $4.5 \mu \mathrm{M}$ and $4.7 \mu \mathrm{M}$ for NLaz-ArA and NLaz-PA respectively.

NLaz-PA binding and the previously known Laz-PA binding [40], led us to test whether PA binding was also conserved in ApoD. Figure 4A shows the titration of PA with ApoD, resulting in a calculated $K_{D}$ of $3.3 \mu \mathrm{M}$. These results contrast with a previous report where ApoD does not measurably bind to PA [34]. Technical differences might underlay these results. While we used $295 \mathrm{~nm}$, which selectively excites tryptophan residues, Morais-Cabral et al. [34] used $280 \mathrm{~nm}$, that also excites tyrosine residues. Energy transfer from tyrosine to tryptophan is common and might complicate the interpretation of results.

\subsubsection{Endocannabinoids and ApoD.}

Endogenous cannabinoids, or endocannabinoids (EC), are lipid modulators of synaptic activity and are involved in memory, anxiety, movement and pain. Predominant ECs include anandamide (AEA) and 2-arachidonoylglycerol (2-AG). AEA and 2-AG are both synthesized from membrane AA glycerophospholipid precursors [reviewed by 63].

How ECs are released from cells and how they reach their targets is a major issue in the field. Recent studies have identified intracellular AEA transport proteins, but the question is still open on whether extracellular EC-transporter proteins exist [see 64 for a review]. It has been suggested that Lipocalins, due to their lipid binding pocket, could be responsible for EC mobilization inside the brain [65]. Three Lipocalins are normally expressed in the human brain: L-PGDS, RBP and ApoD. RBP and PGDS ligands are well known and their functions have been established. ApoD appears therefore as a likely candidate for EC binding. NLaz is also a brain Lipocalin, but the classical cannabinoid receptors are absent in the Drosophila brain [66].

These results, along with ApoD binding to the EC precursor AA [34, 35], point to this Lipocalin as an endocannabinoid carrier. We tested whether ApoD binds to 2-AG or AEA, and found measurable binding with AEA $\left(K_{D} 1.6 \mu \mathrm{M}\right)($ Fig. $4 \mathrm{D}$, E). This is the 
first experimental evidence showing an interaction between ApoD and the endocannabinoid system. This selective binding profile of ApoD is particularly noteworthy, and future in vivo experiments with ApoD-KO and ApoD-overexpressor mice might confirm a physiological role for ApoD in the endocannabinoid system.

\subsubsection{NLaz binding to Drosophila pheromones.}

Pheromones are bioactive lipids involved in the regulation of courtship behavior and mating. NLaz null mutant (NLaz-KO) flies exhibit a dramatically low courtship index compared to their isogenic controls [23, 27]. As a first step to understand the mechanistic origin of this phenotype, we tested whether NLaz is able to bind Drosophila pheromones.

11-cis-vaccenyl acetate (cVA) is the most studied Drosophila pheromone, and acts selectively through the Or67b odorant receptor in both sexes [67]. LUSH is the extracellular carrier for $\mathrm{cVA}$ and undergoes a conformational change that triggers $\mathrm{cVA}$ release and binding to the Or67b receptor [68]. We tested NLaz as an alternative pheromone-binding protein for cVA, but did not detect significant binding (Fig. 3E).

Other Drosophila cuticular sex pheromones are unsaturated long-chain hydrocarbons synthesized from fatty acid precursors. PA, a ligand of NLaz, is also the substrate of Desat1, a PA desaturase that triggers an enzymatic pheromone synthesis pathway [69].

Drosophila hydrocarbon pheromones are dimorphic: monoene hydrocarbons are predominant in males, and dienes in females [70]. We tested whether NLaz is able to bind two of the major Drosophila pheromones: the 7-tricosene (7-T, 23:1) and 7,11heptacosadiene $(7,11-\mathrm{HD}, 27: 2)$. Figure $3 \mathrm{C}$ shows positive binding of NLaz to monoene 7-T, whereas no binding was detected with the diene 7,11-HD (Fig. 3D). The estimated $K_{D}$ of 7-T binding to NLaz is $4.5 \mu \mathrm{M}$. 7-T increases Drosophila female sexual receptivity, making flies to mate faster and more often with males that have higher levels of 7-T, while low levels of 7-T reduces courtship success [71]. We propose that NLaz could be acting as a pheromone carrier protein for 7-T, in a way similar to the LUSH-cVA pair [68].

\subsection{ApoD-RA interaction is required for neuronal differentiation in culture.}

Finally, we assayed whether any of the binding properties of the ApoD-related Lipocalins have direct in vivo consequences. For that purpose, we chose a neuronal cell model (SH-SY5Y and M17 cell lines), and RA as a well-defined common ligand for these proteins.

RA has a crucial role during brain development, being required to induce early progenitors to adopt a neural fate. During embryogenesis there is a requirement for a precise timing of exposure to RA and a coordinated pattern of expression of retinoic acid receptors (RARs) [see 43 for a general review].

SH-SY5Y is a human neuroblastoma cell line that has been established as a model of neuronal differentiation $[72,73]$. Under RA treatment SH-SY5Y cells develop long processes, and cells with processes longer than $50 \mu \mathrm{m}$ have been considered as morphologically differentiated [72]. The presence of at least $1 \%$ serum [74] or exogenous albumin is required to obtain differentiation of SH-SY5Y cells by RA [75]. On the contrary, SH-SY5Y cells quickly enter the apoptosis pathway under serum deprivation [76]. 
ApoD plays an active role during nervous system development, where it is mainly expressed by glial cells, but is also present in neurons during development [8-10] at times when neurite growth is taking place. ApoD has been previously related with cell differentiation. Lopez-Boado el al. [77] first described that human breast cancer T-47D cells differentiate under RA treatment. In these conditions T-47D cells accumulate lipid droplets and increase ApoD levels, both at mRNA and protein levels. This process is RAR $\alpha$-dependent [78]. Moreover, Kosacka et al. [30,50] have shown that ApoD might act as a neurotrophic adipokine and exerts differentiating and synaptogenic effects in dorsal root ganglion neurons. On the other hand, when SH-SY5Y cells are transfected with p 73, a transcription factor of the p53 family involved in neuronal differentiation, cells differentiate to mature neurons in an ApoD-dependent manner [79].

Initially, we confirmed that SH-SY5Y extend neurites under standard differentiation conditions (Fig. 5B and 5C). When serum was removed from the medium, cell survival decreased dramatically and no differentiation was achieved even in the presence of RA, ApoD, NLaz or Laz (Fig. 5E-F and 6A-B). However, cells do differentiate and develop numerous long processes if we add ApoD preloaded with RA (Fig. 5G) in the absence of serum. These results show that ApoD is necessary and sufficient to allow for RA differentiating activity.

To validate as general the effects of ApoD-RA on neuronal differentiation, we used another human neuroblastoma cell line, M17, and obtained identical results (Fig. S5 and S6B).

Thus, ApoD loaded with RA produces neuronal differentiation and promotes neuritogenesis even under serum deprivation conditions. Since RA solubility in water is limited, we propose that ApoD could be acting as a carrier protein for RA. Astrocytes act as a source of ApoD [26] and also provide RA and contribute to RA homeostasis [80]. ApoD could therefore deliver, or make available, RA from astrocytes to neuronal precursors in vivo.

Finally, we tested whether NLaz or Laz can also mediate the differentiation effect of RA on SH-SY5Y cells. RA-preloaded NLaz also induces neuronal differentiation, but in a less efficient way than ApoD-RA or serum-RA (Fig. 6C and E). However, the lengths of processes produced by NLaz-RA are similar to those under ApoD-RA or serum-RA treatment (Fig. S6A). On the other hand, SH-SY5Y cells treated with LazRA do not show a different number of processes than control cells (Fig. 6D and E).

In summary, SH-SY5Y cells treated with ApoD or NLaz, but not Laz, preloaded with RA are able to survive and differentiate in serum free medium, developing long processes. These results indicate that not every RA binding protein is able to trigger the differentiation program. Since NLaz-RA is not as successful as ApoD-RA in triggering SH-SY5Y differentiation, our results also suggests that a specific interaction, perhaps with an ApoD receptor, is further required to obtain the full effect. NLaz or Laz interaction with this putative receptor in mammalian cells might not be as favorable.

In spite of the relevance of RA during early morphogenesis and nervous system development, and the fact that we find an ApoD-RA functional interaction, ApoD null mice do not have major developmental problems and reach adulthood with apparent normality [21]. They show, however, defects in locomotor and spatial memory tests [21 and unpublished observations] revealing a certain extent of wiring defects. Since RA can in principle also bind to other Lipocalins present in the nervous system [e.g. 81, 82], functional redundancy might explain the ability of the ApoD-KO mice to complete their development without major alterations. 


\subsection{ApoD and its insect related Lipocalins: conservation and divergence of ligand binding properties.}

Many studies have shown that human ApoD, Drosophila NLaz and Schistocerca Laz share functional properties in the organism: regulation of lifespan, lipid and carbohydrate metabolism control and protection against oxidative stress or starvation $[21,22,26,27,55]$. This work extends the knowledge of ligand binding for ApoD and Laz, and presents an extensive ligand screening for NLaz.

Our ligand-binding studies show that ApoD-NLaz-Laz similarities also extend to their biochemical and lipid binding properties. Homology modeling of the structures of Laz and NLaz (Fig. 7A) and a comparison of their sequences (Fig. 7C) show the presence of predicted ligand binding sites, broadly consistent with the conserved structures of Lipocalins and the ligand specificities reported here. Some of the ligands for which we have proven a positive interaction are shown at the same scale, either in full or as part of their molecular structure with a configuration most compatible with the ligand binding cavities (Fig. 7B).

The modeled calyces of the insect Laz and NLaz are somewhat smaller in total volume and with moderately greater polar character than the ApoD calyx. As most Lipocalins, NLaz binds retinol and RA. Besides, fatty acids and SM are ligands common to ApoD and its insect related Lipocalins. Whether the SM polar head (shown in Fig. 7B) or its acyl groups are the main interacting moieties in this ligand remains to be resolved. We can predict that these common ligands can be related with in vivo common functions of ApoD, NLaz and Laz. In addition, a set of species-specific ligands are first described here. AEA binding to ApoD and 7-T to NLaz might represent new functions acquired by these Lipocalins during their divergent evolution.

ApoD, NLaz and Laz can be considered as moonlighting proteins, and that pleiotropy is reflected on the high number of putative hydrophobic ligands. Their ligands belong to different groups of lipids, exhibiting different shapes and biochemical properties. In spite of this high variability and apparently promiscuous binding, ApoD, NLaz and Laz show a quite exquisite selectivity between very similar lipidic species. For example, ApoD binds the endocannabinoid AEA, but not 2-AG, and NLaz binds the pheromone 7-T but not 7,11-HD.

Taken together, our ligand screening and the neuronal cell based assay should help us understand how ApoD, NLaz and Laz perform their known biological functions, and how much of each function can be dependent on the particular lipidic ligand involved. Furthermore, the discovery of new putative ligands for these Lipocalins opens the door to explore new functions of ApoD and Lazarillo in vivo.

\section{Materials and Methods}

\subsection{Cell cultures.}

Drosophila S2 cells were cultured as previously described [27]. Human neuroblastoma cell lines SH-SY5Y and M17 were grown at $37^{\circ} \mathrm{C}$ in a saturated humidity atmosphere containing 5\% $\mathrm{CO}_{2}$ in DMEM (Dulbecco's Modified Eagle's Medium, Lonza Iberica, Barcelona, Spain) supplemented with glucose (4.5 g/l), heatinactivated 10\% FBS (fetal bovine serum, Lonza Iberica), 1\% L-Glutamine, antibiotics (penicillin, $10 \mathrm{U} / \mu \mathrm{l}$; streptomycin, $10 \mu \mathrm{g} / \mu \mathrm{l}$; amphotericin B, $25 \mu \mathrm{g} / \mathrm{ml}$; Lonza Iberica), and $1 \%$ non-essential amino acids (NEAA, Lonza Iberica). The culture medium was replaced twice a week, and cells were subcultured at $90 \%$ confluence. 


\subsection{Cloning and protein purification of NLaz, Laz and ApoD.}

The NLaz full-length cDNA, translating into residues 1-224 (CG33126, Uniprot reference Q9NAZ4, FlyBase entry FBgn0053126), and a fragment of Lazarillo cDNA encoding a 192 residue fragment missing the GPI signal peptide (Uniprot reference P49291), were subcloned into the pRmHa3 vector using EcoRI and NotI sites. This system expresses the cloned sequences under the control of an inducible Drosophila metallothionein promoter and incorporates a $\mathrm{C}$-terminal poly-histidine sequence allowing for protein purification from conditioned medium.

NLaz-pRmHa3 or Lazarillo-pRmHa3 plasmids were co-transfected with a pCoBlast selection vector (conferring blasticidin resistance) into Drosophila S2 cells. Protein expression induction and purification were performed as previously described [27], adding two rounds of size exclusion chromatography purification after the initial metal affinity chromatography (Fig. S1B-D).

Bacterial recombinant Laz was purified from the periplasmic space of $E$. coli as previously described [40]. Human ApoD (hApoD) was purified from breast cystic fluid (Fig. S1A and S1D) by ion-exchange chromatography (UNO-Q, BioRad, Hercules, CA, USA) in $25 \mathrm{mM}$ Tris $\mathrm{pH} 8.0$ followed by size exclusion chromatography (P60, BioRad) in $50 \mathrm{mM}$ Tris $\mathrm{pH} 8.0,75 \mathrm{mM} \mathrm{NaCl}$. Protein purity was determined by SDS-PAGE and integrated optical density of the protein bands stained with Coomassie was measured, resulting in $91 \%$ purity for ApoD and $>98 \%$ purity for NLaz and Laz (Fig. S1).

\subsection{Ligand binding assays by tryptophan fluorescence titration.}

Fluorescence measurements were conducted with a Shimadzu RF-5301PC spectrofluorometer in a quartz cuvette (Hellma, Müllheim, Germany, 105.251-QS, 3 mm-path length). Temperature was held at $22 \pm 0.1^{\circ} \mathrm{C}$. The excitation wavelength was $295 \mathrm{~nm}$ (selective for tryptophan residues). Emission was recorded from 327 to $400 \mathrm{~nm}$ with slit width set at $5 \mathrm{~nm}$. The peak observed at $340 \mathrm{~nm}$ was measured and used to calculate the apparent equilibrium dissociation constant $\left(K_{D}\right)$. Data analysis was performed with the RFPC software package (Shimadzu, Kyoto, Japan). NLaz and the control protein $\alpha 1$-microglobulin were diluted to a concentration of $1 \mu \mathrm{M}$ in $10 \mathrm{mM}$ phosphate buffer, $150 \mathrm{mM} \mathrm{NaCl}, 1 \mathrm{mM}$ EDTA at $\mathrm{pH}$ 7.0. Laz and hApoD were diluted to a concentration of $0.5 \mu \mathrm{M}$ in the same buffer, so that the starting intrinsic fluorescence of all proteins lies within the same dynamic range of measurement. The ligands tested were: retinoic acid, retinol, non-esterified cholesterol, ergosterol, $\beta$ estradiol, palmitic acid (Sigma St. Louis, MO, USA ); palmitoyl-sphingomyelin, arachidic acid, 7(z)-tricosene, 7(z),11(z)-heptacosadiene, 11-cis-vaccenyl acetate, palmitoyl-linoleyl-phosphoethanolamine, 2-arachidonylglycerol, anandamide (Cayman, Tallinn, Estonia); and 20-hydroxyecdysone (Santa Cruz Biotechnology, Dallas, TX, USA). All ligands were dissolved in N,N-dimethylformamide (DMF; Sigma) to make concentrated stock solutions within their solubility range. A four step titration was performed, adding each time $1 \mu \mathrm{l}$ aliquots of the ligand to a $100 \mu \mathrm{l}$ volume of protein solution. The mixtures were equilibrated for $3 \mathrm{~min}$ in the dark before the fluorescence was recorded.

The fluorescence spectrum in the presence of a ligand was subtracted from a DMF baseline obtained by titration of the protein with the same amounts of carrier without ligand. The corrected fluorescence at $340 \mathrm{~nm}$ versus ligand concentration was fitted as previously described $[37,40] . K_{D}$ was calculated under the assumption of a single binding site, consistent with known Lipocalin structural properties, regardless of whether ligand binding enhances or quenches tryptophan fluorescence. A tryptophan residue conserved along the entire Lipocalin family is located at the binding pocket 
surface, and is predicted to be the residue responsible for most of the intrinsic fluorescence changes observed. The specificity of protein fluorescence variation was tested by performing the assay with the protein denatured with $5 \mathrm{M}$ guanidine hydrochloride in the same buffer for $21 \mathrm{~h}$ before measurement (Fig. S2).

\subsection{Neuronal cell line differentiation assay.}

SH-SY5Y or M17 cells were seeded at $20,000 / \mathrm{cm}^{2}$ in 24 well plates (Nunc) with standard medium. After $12 \mathrm{~h}$, the medium was replaced. New medium was added with or without serum or additives (protein, ligand or equimolar amounts of both protein and ligand). Retinoic acid and retinol stock solutions were prepared in ethanol and diluted in medium to a final concentration of 1 or $10 \mu \mathrm{M}$. The carrier concentration was kept at $0.1 \%(\mathrm{v} / \mathrm{v})$. The concentrations of hApoD, NLaz and Lazarillo were measured by MicroBCA-assay (Pierce, Rockford, IL, USA) and the proteins were diluted in sterile PBS to prepare the working protein stocks. Equimolar protein-ligand mixtures were incubated in the dark at room temperature for five minutes to allow for complexation before addition to cells. SH-SY5Y cultures were analyzed after 4-6 days of growth in the presence of additives, and M17 cultures were analyzed after 3-4 days of growth. Photomicrographs were analyzed with NISelements software (Nikon) with morphological differentiation determined by the number of neurites (processes longer than $50 \mu \mathrm{m})$ present per field [72].

\subsection{Statistical analysis.}

Statistical analyses were performed with Sigma Plot software (v 11.0). KruskalWallis one-way ANOVA on Ranks followed by Dunn's multiple comparison post-hoc tests were used in the analysis of neurite promoting activity of Lipocalins. A p-value $<$ 0.05 , marked with an asterisk, was used as a threshold for significant changes. Two asterisks were used for $p<0.001$.

\section{Acknowledgments}

We thank Dr. Bo Åkerström who kindly donated the $\alpha$-1-microglobulin protein purified from a bacterial expression system. We thank Dr. Sonia Gallego for SH-SY5Y cells, and Dr. R. Bajo-Grañeras and Dr. Angel Cedazo for M17 cell lines donation. E. MartinTejedor provided technical assistance throughout the project.

Grant support. This work was supported by grants to M.D.G. and D.S. (Junta de Castilla y León (JCyL) grant VA180A11-2, and Ministerio de Ciencia e Innovación (MICINN) grants BFU2008-01170 and BFU2011-23978). M.R. was supported by a JCyL fellowship to young researchers (call\#EDU/1708/2008).

\section{References}

1. Newcomer ME \& Ong DE (2006) Retinol Binding Protein and Its Interaction with Transthyretin. In Lipocalins (Åkerström B, Borregaard N, Flower DR \& Salier JP, $\mathrm{ed}^{\wedge}$ eds). Landes Bioscience, Georgetown, Texas. 
2. Sevvana M, Ahnstrom J, Egerer-Sieber C, Lange HA, Dahlback B \& Muller YA (2009) Serendipitous fatty acid binding reveals the structural determinants for ligand recognition in apolipoprotein M. J Mol Biol 393, 920-936.

3. Urade Y, Eguchi N \& Osamu Hayaishi O (2006) Lipocalin-Type Prostaglandin D Synthase as an Enzymic Lipocali. In Lipocalins (Åkerström B, Borregaard N, Flower DR \& Salier JP, ed^eds). Landes Bioscience, Georgetown, Texas.

4. Flower DR, North AC \& Sansom CE (2000) The lipocalin protein family: structural and sequence overview. Biochim Biophys Acta 1482, 9-24.

5. Rassart E, Bedirian A, Do Carmo S, Guinard O, Sirois J, Terrisse L \& Milne R (2000) Apolipoprotein D. Biochim Biophys Acta 1482, 185-198.

6. McConathy WJ \& Alaupovic P (1976) Studies on the isolation and partial characterization of apolipoprotein D and lipoprotein D of human plasma. Biochemistry 15, 515-520.

7. Goessling W \& Zucker SD (2000) Role of apolipoprotein D in the transport of bilirubin in plasma. Am J Physiol Gastrointest Liver Physiol 279, G356-365.

8. $\quad$ Ong WY, Lau CP, Leong SK, Kumar U, Suresh S \& Patel SC (1999) Apolipoprotein D gene expression in the rat brain and light and electron microscopic immunocytochemistry of apolipoprotein D expression in the cerebellum of neonatal, immature and adult rats. Neuroscience 90, 913-922.

9. Sanchez D, Ganfornina MD \& Martinez S (2002) Expression pattern of the lipocalin apolipoprotein D during mouse embryogenesis. Mech Dev 110, 225-229.

10. Ganfornina MD, Sanchez D, Pagano A, Tonachini L, Descalzi-Cancedda F \& Martinez S (2005) Molecular characterization and developmental expression pattern of the chicken apolipoprotein D gene: implications for the evolution of vertebrate lipocalins. Dev Dyn 232, 191-199.

11. Kim WS, Wong J, Weickert CS, Webster MJ, Bahn S \& Garner B (2009) Apolipoprotein-D expression is increased during development and maturation of the human prefrontal cortex. J Neurochem 109, 1053-1066.

12. Loerch PM, Lu T, Dakin KA, Vann JM, Isaacs A, Geula C, Wang J, Pan Y, Gabuzda DH, Li C, Prolla TA \& Yankner BA (2008) Evolution of the aging brain transcriptome and synaptic regulation. PLoS One 3, e3329.

13. de Magalhaes JP, Curado J \& Church GM (2009) Meta-analysis of age-related gene expression profiles identifies common signatures of aging. Bioinformatics 25, 875881.

14. Terrisse L, Poirier J, Bertrand P, Merched A, Visvikis S, Siest G, Milne R \& Rassart E (1998) Increased levels of apolipoprotein D in cerebrospinal fluid and hippocampus of Alzheimer's patients. J Neurochem 71, 1643-1650. 
15. Ordonez C, Navarro A, Perez C, Astudillo A, Martinez E \& Tolivia J (2006)

Apolipoprotein D expression in substantia nigra of Parkinson disease. Histol

Histopathol 21, 361-366.

16. Thomas EA, Dean B, Pavey G \& Sutcliffe JG (2001) Increased CNS levels of apolipoprotein D in schizophrenic and bipolar subjects: implications for the pathophysiology of psychiatric disorders. Proc Natl Acad Sci U S A 98, 4066-4071.

17. Kroksveen AC, Aasebo E, Vethe H, Van Pesch V, Franciotta D, Teunissen CE, Ulvik RJ, Vedeler C, Myhr KM, Barsnes H \& Berven FS (2012) Discovery and initial verification of differentially abundant proteins between multiple sclerosis patients and controls using iTRAQ and SID-SRM. J Proteomics.

18. Suresh S, Yan Z, Patel RC, Patel YC \& Patel SC (1998) Cellular cholesterol storage in the Niemann-Pick disease type $C$ mouse is associated with increased expression and defective processing of apolipoprotein D. J Neurochem 70, 242-251.

19. Ganfornina MD, Gutierrez G, Bastiani M \& Sanchez D (2000) A phylogenetic analysis of the lipocalin protein family. Mol Biol Evol 17, 114-126.

20. Sanchez D, Ganfornina MD, Gutierrez G \& Marin A (2003) Exon-intron structure and evolution of the Lipocalin gene family. Mol Biol Evol 20, 775-783.

21. Ganfornina MD, Do Carmo S, Lora JM, Torres-Schumann S, Vogel M, Allhorn M, González C, Bastiani MJ, Rassart E \& Sanchez D (2008) Apolipoprotein D is involved in the mechanisms regulating protection from oxidative stress. Aging Cell 7, 506-515.

22. Hull-Thompson J, Muffat J, Sanchez D, Walker DW, Benzer S, Ganfornina MD \& Jasper H (2009) Control of metabolic homeostasis by stress signaling is mediated by the lipocalin NLaz. PLoS Genet 5, e1000460.

23. Ruiz M, Sanchez D, Canal I, Acebes A \& Ganfornina MD (2011) Sex-dependent modulation of longevity by two Drosophila homologues of human Apolipoprotein D, GLaz and NLaz. Exp Gerontol 46, 579-589.

24. Do Carmo S, Fournier D, Mounier C \& Rassart E (2009) Human apolipoprotein $\mathrm{D}$ overexpression in transgenic mice induces insulin resistance and alters lipid metabolism. Am J Physiol Endocrinol Metab 296, E802-811.

25. Jimenez-Palomares M, Cozar-Castellano I, Ganfornina MD, Sanchez D \& Perdomo G (2011) Genetic deficiency of apolipoprotein D in the mouse is associated with nonfasting hypertriglyceridemia and hyperinsulinemia. Metabolism 60, 1767-1774.

26. Bajo-Graneras R, Ganfornina MD, Martin-Tejedor E \& Sanchez D (2011) Apolipoprotein D mediates autocrine protection of astrocytes and controls their reactivity level, contributing to the functional maintenance of paraquat-challenged dopaminergic systems. Glia 59, 1551-1566.

27. Ruiz M, Wicker-Thomas C, Sanchez D \& Ganfornina MD (2012) Grasshopper Lazarillo, a GPI-anchored Lipocalin, increases Drosophila longevity and stress 
resistance, and functionally replaces its secreted homolog NLaz. Insect Biochem Mol Biol 42, 776-789.

28. Zhang Y, Cong Y, Wang S \& Zhang S (2011) Antioxidant activities of recombinant amphioxus (Branchiostoma belcheri) apolipoprotein D. Mol Biol Rep 38, $1847-1851$.

29. Bhatia S, Knoch B, Wong J, Kim WS, Else PL, Oakley AJ \& Garner B (2012) Selective reduction of hydroperoxyeicosatetraenoic acids to their hydroxy derivatives by apolipoprotein D: implications for lipid antioxidant activity and Alzheimer's disease. Biochem J 442, 713-721.

30. Kosacka J, Schroder T, Bechmann I, Kloting N, Nowicki M, Mittag A, Gericke M, Spanel-Borowski K \& Bluher M (2011) PACAP up-regulates the expression of apolipoprotein D in 3T3-L1 adipocytes. DRG/3T3-L1 co-cultures study. Neurosci Res 69, 8-16.

31. Pearlman WH, Gueriguian JL \& Sawyer ME (1973) A specific progesteronebinding component of human breast cyst fluid. J Biol Chem 248, 5736-5741.

32. Lea OA (1988) Binding properties of progesterone-binding Cyst protein, PBCP. Steroids 52, 337-338.

33. Dilley WG, Haagensen DE, Cox CE \& Wells SA, Jr. (1990) Immunologic and steroid binding properties of the GCDFP-24 protein isolated from human breast gross cystic disease fluid. Breast Cancer Res Treat 16, 253-260.

34. Morais Cabral JH, Atkins GL, Sanchez LM, Lopez-Boado YS, Lopez-Otin C \& Sawyer L (1995) Arachidonic acid binds to apolipoprotein D: implications for the protein's function. FEBS Lett 366, 53-56.

35. Vogt M \& Skerra A (2001) Bacterially produced apolipoprotein D binds progesterone and arachidonic acid, but not bilirubin or E-3M2H. J Mol Recognit 14, 7986.

36. Eichinger A, Nasreen A, Kim HJ \& Skerra A (2007) Structural insight into the dual ligand specificity and mode of high density lipoprotein association of apolipoprotein D. J Biol Chem 282, 31068-31075.

37. Breustedt DA, Schonfeld DL \& Skerra A (2006) Comparative ligand-binding analysis of ten human lipocalins. Biochim Biophys Acta 1764, 161-173.

38. Zeng C, Spielman AI, Vowels BR, Leyden JJ, Biemann K \& Preti G (1996) A human axillary odorant is carried by apolipoprotein D. Proc Natl Acad Sci U S A 93, 6626-6630.

39. Patel RC, Lange D, McConathy WJ, Patel YC \& Patel SC (1997) Probing the structure of the ligand binding cavity of lipocalins by fluorescence spectroscopy.

Protein Eng 10, 621-625. 
40. Sanchez D, Ortega-Cubero S, Åkerström B, Herrera M, Bastiani MJ \& Ganfornina MD (2008) Molecular interactions of the neuronal GPI-anchored lipocalin Lazarillo. Journal of Molecular Recognition 21, 313-323.

41. Goodman DS (1980) Plasma retinol-binding protein. Ann N Y Acad Sci 348, 378-390.

42. Biesalski HK, Frank J, Beck SC, Heinrich F, Illek B, Reifen R, Gollnick H, Seeliger MW, Wissinger B \& Zrenner E (1999) Biochemical but not clinical vitamin A deficiency results from mutations in the gene for retinol binding protein. Am J Clin Nutr 69, 931-936.

43. Rhinn M \& Dolle $\mathrm{P}$ (2012) Retinoic acid signalling during development. Development 139, 843-858.

44. Nowickyj SM, Chithalen JV, Cameron D, Tyshenko MG, Petkovich M, Wyatt GR, Jones G \& Walker VK (2008) Locust retinoid X receptors: 9-Cis-retinoic acid in embryos from a primitive insect. Proc Natl Acad Sci U S A 105, 9540-9545.

45. Sanchez D, Ganfornina MD \& Bastiani MJ (2000) Lazarillo, a neuronal lipocalin in grasshoppers with a role in axon guidance. Biochim Biophys Acta 1482, 102-109.

46. McConathy WJ \& Alaupovic P (1973) Isolation and partial characterization of apolipoprotein D: a new protein moiety of the human plasma lipoprotein system. FEBS Lett 37, 178-182.

47. Blanco-Vaca F, Via DP, Yang CY, Massey JB \& Pownall HJ (1992)

Characterization of disulfide-linked heterodimers containing apolipoprotein D in human plasma lipoproteins. J Lipid Res 33, 1785-1796.

48. Akerström B, Borregaard N, Flover D \& Salier J (2006) Lipocalins, Georgetown, Texas.

49. Perdomo G, Kim DH, Zhang T, Qu S, Thomas EA, Toledo FG, Slusher S, Fan Y, Kelley DE \& Dong HH (2010) A role of apolipoprotein D in triglyceride metabolism. J Lipid Res 51, 1298-1311.

50. Kosacka J, Gericke M, Nowicki M, Kacza J, Borlak J \& Spanel-Borowski K (2009) Apolipoproteins D and E3 exert neurotrophic and synaptogenic effects in dorsal root ganglion cell cultures. Neuroscience 162, 282-291.

51. He X, Jittiwat J, Kim JH, Jenner AM, Farooqui AA, Patel SC \& Ong WY (2009) Apolipoprotein D modulates F2-isoprostane and 7-ketocholesterol formation and has a neuroprotective effect on organotypic hippocampal cultures after kainate-induced excitotoxic injury. Neurosci Lett 455, 183-186.

52. Rietveld A, Neutz S, Simons K \& Eaton S (1999) Association of sterol- and glycosylphosphatidylinositol-linked proteins with Drosophila raft lipid microdomains. $J$ Biol Chem 274, 12049-12054. 
53. Vance DE \& Vance JE (2008) Biochemistry of Lipids, Lipoproteins and Membranes, 5th edn. Elsevier.

54. Nagao K, Takahashi K, Hanada K, Kioka N, Matsuo M \& Ueda K (2007) Enhanced apoA-I-dependent cholesterol efflux by ABCA1 from sphingomyelindeficient Chinese hamster ovary cells. J Biol Chem 282, 14868-14874.

55. Ganfornina MD, Do Carmo S, Martínez E, Tolivia J, Navarro A, Rassart E \& Sanchez D (2010) ApoD, a glia-derived apolipoprotein, is required for peripheral nerve functional integrity and a timely response to injury. Glia 58, 1320-1334.

56. Elsoe S, Ahnstrom J, Christoffersen C, Hoofnagle AN, Plomgaard P, Heinecke JW, Binder CJ, Bjorkbacka H, Dahlback B \& Nielsen LB (2012) Apolipoprotein M binds oxidized phospholipids and increases the antioxidant effect of HDL.

Atherosclerosis 221, 91-97.

57. Soiland H, Soreide K, Janssen EA, Korner H, Baak JP \& Soreide JA (2007) Emerging concepts of apolipoprotein D with possible implications for breast cancer. Cell Oncol 29, 195-209.

58. Lambert J, Provost PR, Marcel YL \& Rassart E (1993) Structure of the human apolipoprotein D gene promoter region. Biochim Biophys Acta 1172, 190-192.

59. Truman JW (2005) Hormonal control of insect ecdysis: endocrine cascades for coordinating behavior with physiology. Vitam Horm 73, 1-30.

60. Fraser T, Tayler H \& Love S (2010) Fatty acid composition of frontal, temporal and parietal neocortex in the normal human brain and in Alzheimer's disease.

Neurochem Res 35, 503-513.

61. Fabelo N, Martin V, Santpere G, Marin R, Torrent L, Ferrer I \& Diaz M (2011) Severe alterations in lipid composition of frontal cortex lipid rafts from Parkinson's disease and incidental Parkinson's disease. Mol Med 17, 1107-1118.

62. Yao JK, Leonard S \& Reddy RD (2000) Membrane phospholipid abnormalities in postmortem brains from schizophrenic patients. Schizophr Res 42, 7-17.

63. Kano M, Ohno-Shosaku T, Hashimotodani Y, Uchigashima M \& Watanabe M (2009) Endocannabinoid-mediated control of synaptic transmission. Physiol Rev 89, 309-380.

64. Fowler CJ (2012) Anandamide uptake explained? Trends Pharmacol Sci 33, 181-185.

65. Piomelli D (2003) The molecular logic of endocannabinoid signalling. Nat Rev Neurosci 4, 873-884.

66. McPartland J, Di Marzo V, De Petrocellis L, Mercer A \& Glass M (2001)

Cannabinoid receptors are absent in insects. J Comp Neurol 436, 423-429.

67. Kurtovic A, Widmer A \& Dickson BJ (2007) A single class of olfactory neurons mediates behavioural responses to a Drosophila sex pheromone. Nature 446, 542-546. 
68. Laughlin JD, Ha TS, Jones DN \& Smith DP (2008) Activation of pheromonesensitive neurons is mediated by conformational activation of pheromone-binding protein. Cell 133, 1255-1265.

69. Labeur C, Dallerac R \& Wicker-Thomas C (2002) Involvement of desat1 gene in the control of Drosophila melanogaster pheromone biosynthesis. Genetica 114, 269274.

70. Ferveur JF (2005) Cuticular hydrocarbons: their evolution and roles in Drosophila pheromonal communication. Behav Genet 35, 279-295.

71. Grillet M, Dartevelle L \& Ferveur JF (2006) A Drosophila male pheromone affects female sexual receptivity. Proc Biol Sci 273, 315-323.

72. Pahlman S, Ruusala AI, Abrahamsson L, Mattsson ME \& Esscher T (1984) Retinoic acid-induced differentiation of cultured human neuroblastoma cells: a comparison with phorbolester-induced differentiation. Cell Differ 14, 135-144.

73. Encinas M, Iglesias M, Liu Y, Wang H, Muhaisen A, Cena V, Gallego C \& Comella JX (2000) Sequential treatment of SH-SY5Y cells with retinoic acid and brainderived neurotrophic factor gives rise to fully differentiated, neurotrophic factordependent, human neuron-like cells. J Neurochem 75, 991-1003.

74. Lopes FM, Schroder R, da Frota ML, Jr., Zanotto-Filho A, Muller CB, Pires AS, Meurer RT, Colpo GD, Gelain DP, Kapczinski F, Moreira JC, Fernandes Mda C \& Klamt F (2010) Comparison between proliferative and neuron-like SH-SY5Y cells as an in vitro model for Parkinson disease studies. Brain Res 1337, 85-94.

75. Nordin-Andersson M, Walum E, Kjellstrand P \& Forsby A (2003) Acrylamideinduced effects on general and neurospecific cellular functions during exposure and recovery. Cell Biol Toxicol 19, 43-51.

76. Andoh T, Chock PB \& Chiueh CC (2002) The roles of thioredoxin in protection against oxidative stress-induced apoptosis in SH-SY5Y cells. J Biol Chem 277, $9655-$ 9660 .

77. Lopez-Boado YS, Tolivia J \& Lopez-Otin C (1994) Apolipoprotein D gene induction by retinoic acid is concomitant with growth arrest and cell differentiation in human breast cancer cells. J Biol Chem 269, 26871-26878.

78. Lopez-Boado YS, Klaus M, Dawson MI \& Lopez-Otin C (1996) Retinoic acidinduced expression of apolipoprotein $\mathrm{D}$ and concomitant growth arrest in human breast cancer cells are mediated through a retinoic acid receptor RARalpha-dependent signaling pathway. J Biol Chem 271, 32105-32111.

79. Sasaki Y, Negishi H, Koyama R, Anbo N, Ohori K, Idogawa M, Mita H, Toyota M, Imai K, Shinomura Y \& Tokino T (2009) p53 family members regulate the expression of the apolipoprotein D gene. J Biol Chem 284, 872-883.

80. Shearer KD, Fragoso YD, Clagett-Dame M \& McCaffery PJ (2012) Astrocytes as a regulated source of retinoic acid for the brain. Glia 60, 1964-1976. 
81. Nagata A, Suzuki Y, Igarashi M, Eguchi N, Toh H, Urade Y \& Hayaishi O (1991) Human brain prostaglandin D synthase has been evolutionarily differentiated from lipophilic-ligand carrier proteins. Proc Natl Acad Sci U S A 88, 4020-4024.

82. Zhang PX, Zhang FR, Xie JJ, Tao LH, Lu Z, Xu XE, Shen J, Xu LY \& Li EM (2012) Expression of NGAL and NGALR in human embryonic, fetal and normal adult tissues. Mol Med Rep 6, 716-722.

83. Holden HM, Rypniewski WR, Law JH \& Rayment I (1987) The molecular structure of insecticyanin from the tobacco hornworm Manduca sexta L. at 2.6 A resolution. EMBO J 6, 1565-1570. 


\section{Figure Legends}

\section{Fig. 1.}

Relative fluorescence intensity of hApoD and its insect homologues NLaz and Laz exposed to increasing concentrations of retinoic acid or retinol. hApoD and Laz protein concentration was $0.5 \mu \mathrm{M}$ in $10 \mathrm{mM}$ phosphate buffer, $150 \mathrm{mM} \mathrm{NaCl}, 1 \mathrm{mM}$ EDTA at pH 7.0. NLaz concentration was $1 \mu \mathrm{M}$ in the same buffer. (A) hApoD-RA; (B) NLazRA; (C) Laz-RA; (D) hApoD-retinol; (E) NLaz-retinol; (F) Laz-retinol. Dots represent the mean of at least 5 independent titrations.

\section{Fig. 2.}

Intrinsic fluorescence changes of hApoD, NLaz and Laz upon incubation with different lipids present in biological membranes. (A) Stable fluorescence of hApoD upon exposure to increasing concentrations of cholesterol. (B) NLaz fluorescence enhancement by ergosterol. (C) Laz fluorescence enhancement by ergosterol. (D-F). Palmitoyl-sphingomyelin enhances the fluorescence of hApoD, NLaz and Laz. Dots represent the mean of at least 3 independent titrations.

\section{Fig. 3.}

Binding assays of hApoD and NLaz with hormones and pheromones. (A) $\beta$-estradiol does not alter the intrinsic fluorescence of hApoD. (B) NLaz does not bind 20hydroxyecdysone. (C) NLaz selectively binds the pheromone 7(z)-tricosene, whereas no interaction was detected for 7(z),11(z)-heptacosadiene (D), or 11-cis-vaccenyl acetate (E). Dots represent the mean of at least independent 3 titrations.

\section{Fig. 4.}

Interaction study of fatty acids and arachidonic acid-related molecules with hApoD and/or NLaz. (A, B) hApoD and NLaz bind palmitic acid. (C) NLaz is also able to bind arachidic acid. (D-E) Binding of ApoD to endocannabinoids. hApoD does not bind 2arachidonyl glycerol (D), but anandamide successfully interacts with hApoD enhancing its fluorescence (E). Dots represent the mean of at least independent 3 titrations.

\section{Fig. 5.}

Physiological role of RA and hApoD in neuronal differentiation. (A) SH-SY5Y cells growing in complete medium (10\% FBS) with ethanol carrier. (B-C) Cells cultured in complete medium after 4-6 days of incubation with $10 \mu \mathrm{M}$ RA (B) or $1 \mu \mathrm{M}$ RA (C) in ethanol. (D) Cells cultured in serum-free medium plus carrier. (E-G) Serum-free medium was supplemented with $1 \mu \mathrm{M}$ RA (E), $1 \mu \mathrm{M}$ hApoD (F) or a mixture of $1 \mu \mathrm{M}$ RA and $1 \mu \mathrm{M}$ hApoD $(\mathrm{G})$. Cell growth was monitored after 4-6 days in culture. $(\mathrm{H})$ Quantification of the number of neurites observed per field. Bars represent the quantification of 8-18 fields per condition from 5-6 independent experiments. KruskalWallis one-way ANOVA on Ranks $(p<0.001)$ followed by Dunn's multiple comparison post-hoc test. In SH-SY5Y cells hApoD is able to substitute for FBS to allow maturation and neurite growth.

\section{Fig. 6.}

Effects of long-term RA and/or NLaz-Laz treatments on SH-SY5Y cell differentiation. Cells grown in serum-free medium containing: (A) $1 \mu \mathrm{M} \mathrm{NLaz}$, (B) $1 \mu \mathrm{M} \mathrm{Laz}$, (C) 1 $\mu \mathrm{M}$ RA plus $1 \mu \mathrm{M}$ NLaz or (D) $1 \mu \mathrm{M}$ RA plus $1 \mu \mathrm{M}$ Laz. (E) Quantification of neurites observed per field normalized by the number of cells per field in each condition. Bars 
represent the quantification of 8-16 photographs from 4-5 independent experiments. Kruskal-Wallis one-way ANOVA on Ranks $(p<0.001)$ followed by Dunn's multiple comparison post-hoc test. NLaz and RA are able to induce some degree of differentiation on SH.SY5Y cells, whereas Laz does not produce any effect.

Fig. 7.

Sequence and structure comparisons of ApoD with NLaz and Laz. (A) Cutaway views of the molecular surfaces of the crystal structure (PDB accession code 2HZQ, [36]) of ApoD and the homology models of Laz and NLaz are shown, colored by electrostatic potential, highlighting the calyces. Images were prepared with the PyMOL Molecular Graphics System, Version 1.3 (Schrödinger, LLC.). The progesterone ligand of ApoD is shown in a licorice stick representation, colored by atom type. Homology models were generated with SWISS-MODEL using the crystal structure of insecticyanin from the tobacco hornworm Manduca sexta (PDB accession code 1Z24, [83]) as a template. (B) Examples of ligands showing binding to at least one of the three Lipocalins are shown to scale. Either the complete molecule (RA, ergosterol) or parts of it (SM) are shown. (C) Sequence alignment showing ApoD-progesterone contact residues and corresponding amino acids in NLaz and Laz (highlighted in green). The conserved W (also an ApoD-progesterone interacting residue) is highlighted in orange. Structurally conserved regions (SCR) in the Lipocalin family are shown in brackets. $\beta$-strands are indicated by arrows and the $\alpha$-helix by a cylinder. Asterisks mark the conserved cysteines, forming disulfide bonds. 


\section{Supplementary Figure Legends}

Fig. S1.

Expression and purification of hApoD from human breast cystic fluid, and NLaz and Laz from S2 cells. Representative size-exclusion chromatography elution profile of hApoD (P60) (A), NLaz (analytical grade-200 column) (B), and Laz (analytical grade200 column) (C). (D) SDS-PAGE analysis of the purified Lipocalins.

\section{Fig. S2.}

Lack of retinoic acid binding to hApoD (A), NLaz (B) and Laz (C) under denaturing conditions. Binding assays were performed either in the standard buffer or after $21 \mathrm{~h}$ incubation with $5 \mathrm{M}$ guanidine hydrochloride. Lipocalins-RA interaction depends on the protein tertiary structure.

\section{Fig. S3.}

Additional intrinsic fluorescence titration studies. Recombinant Laz purified from $E$. coli reproduces the binding pattern of Laz purified from S2 cells. (A) Laz binds retinoic acid and (B) palmitoyl-sphingomyelin. A different Lipocalin ( $\alpha 1-\mathrm{mg})$ also binds retinol (C), but does not bind palmitoyl-sphingomyelin (D).

Fig. S4.

Additional intrinsic fluorescence titration studies. (D) hApoD, (E) Nlaz and (F) Laz do not bind the phospholipid PLPE.

Fig. S5.

Confirmation of the physiological role of RA and hApoD in M17 cells, a different human neuronal cell line. Morphology changes of M17 cells growing in complete medium with (A) ethanol carrier, and after treatment with (B) $10 \mu \mathrm{M}$ RA or (C) $1 \mu \mathrm{M}$ RA in complete medium. (D) Cells in serum-free medium with ethanol carrier. (E-G) Cell differentiation after 4-6 days of treatment with (E) $1 \mu \mathrm{M}$ RA, (F) $1 \mu \mathrm{M}$ hApoD, or (G) $1 \mu \mathrm{M}$ RA plus $1 \mu \mathrm{M}$ hApoD. (H) Quantification of the number of neurite processes /field normalized by the number of cells/field in each condition. Bars represent the quantification of 10-25 fields per condition from 3 independent experiments. KruskalWallis one-way ANOVA on Ranks $(\mathrm{p}<0.001)$ followed by Dunn's multiple comparison post-hoc test. In M17 cells, hApoD is also able to substitute for serum and to allow maturation and neurite growth.

\section{Fig. S6.}

Quantification of neurite length upon RA-induced differentiation of (A) SH-SY5Y or (B) M17 neuronal cell lines. No changes are observed in the length of neurites between treatments. Kruskal-Wallis one-way ANOVA on Ranks ( $>>0.05)$. 
Fig. 1
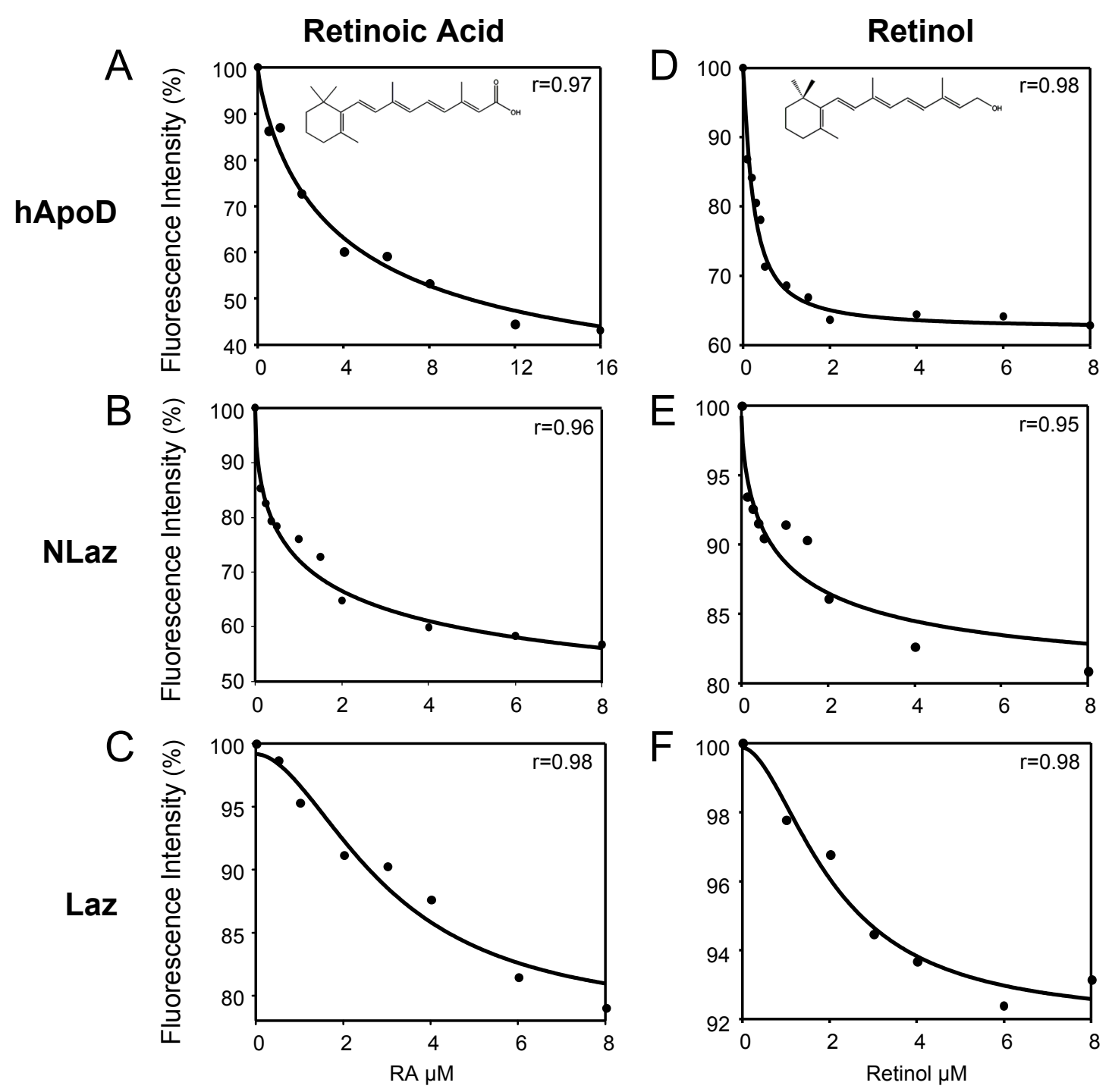
Fig. 2
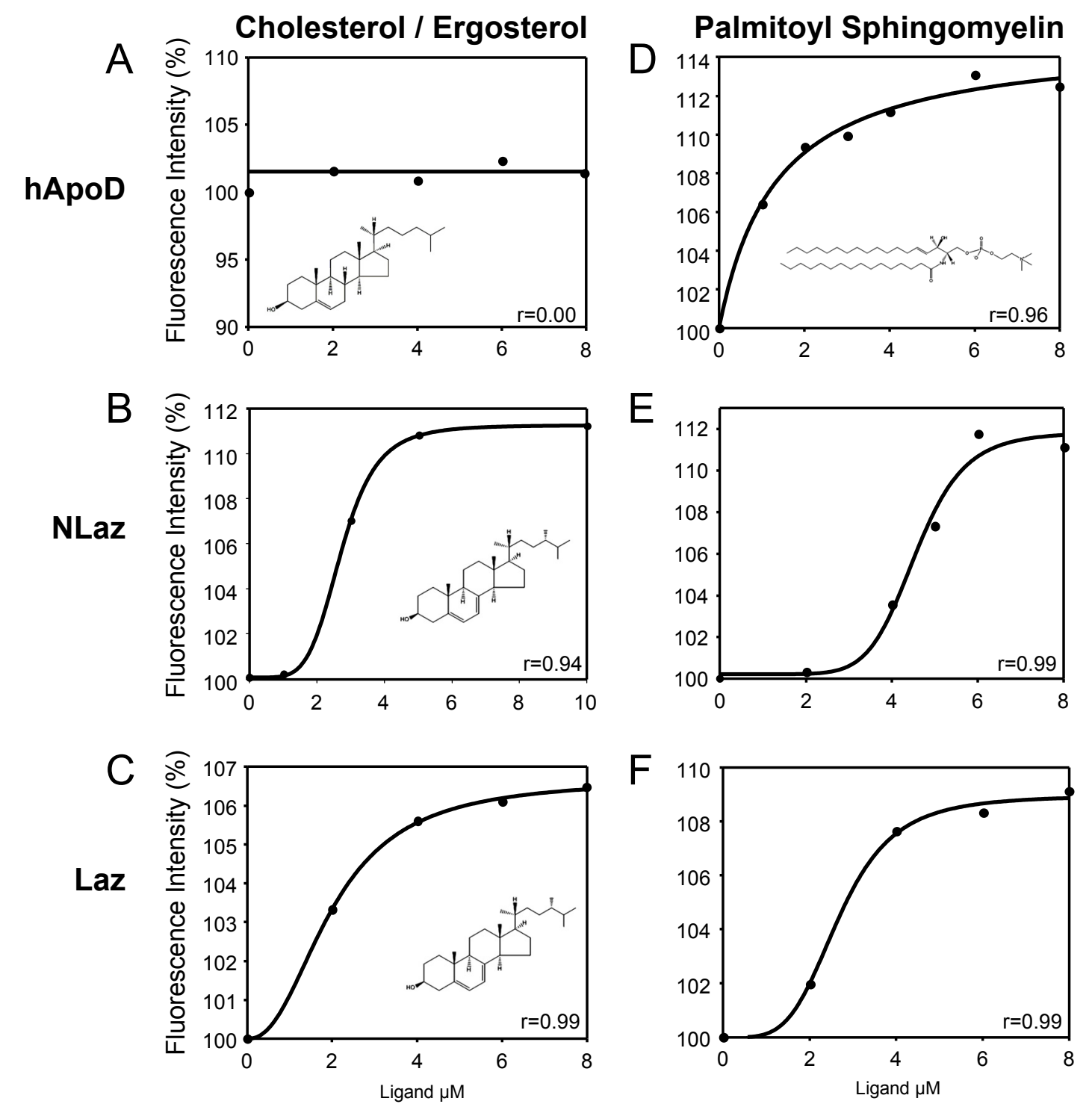
Fig. 3
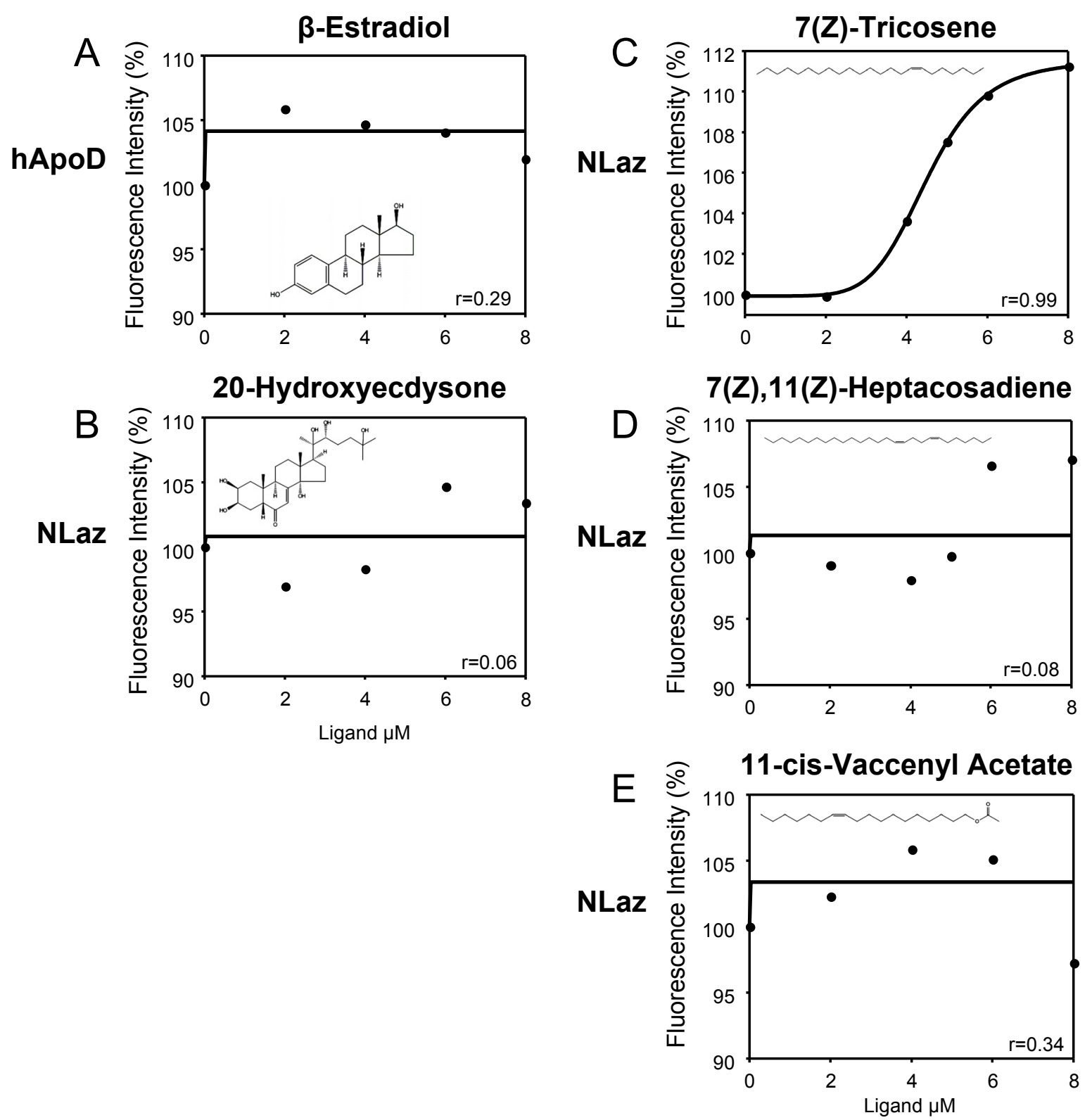
Fig. 4
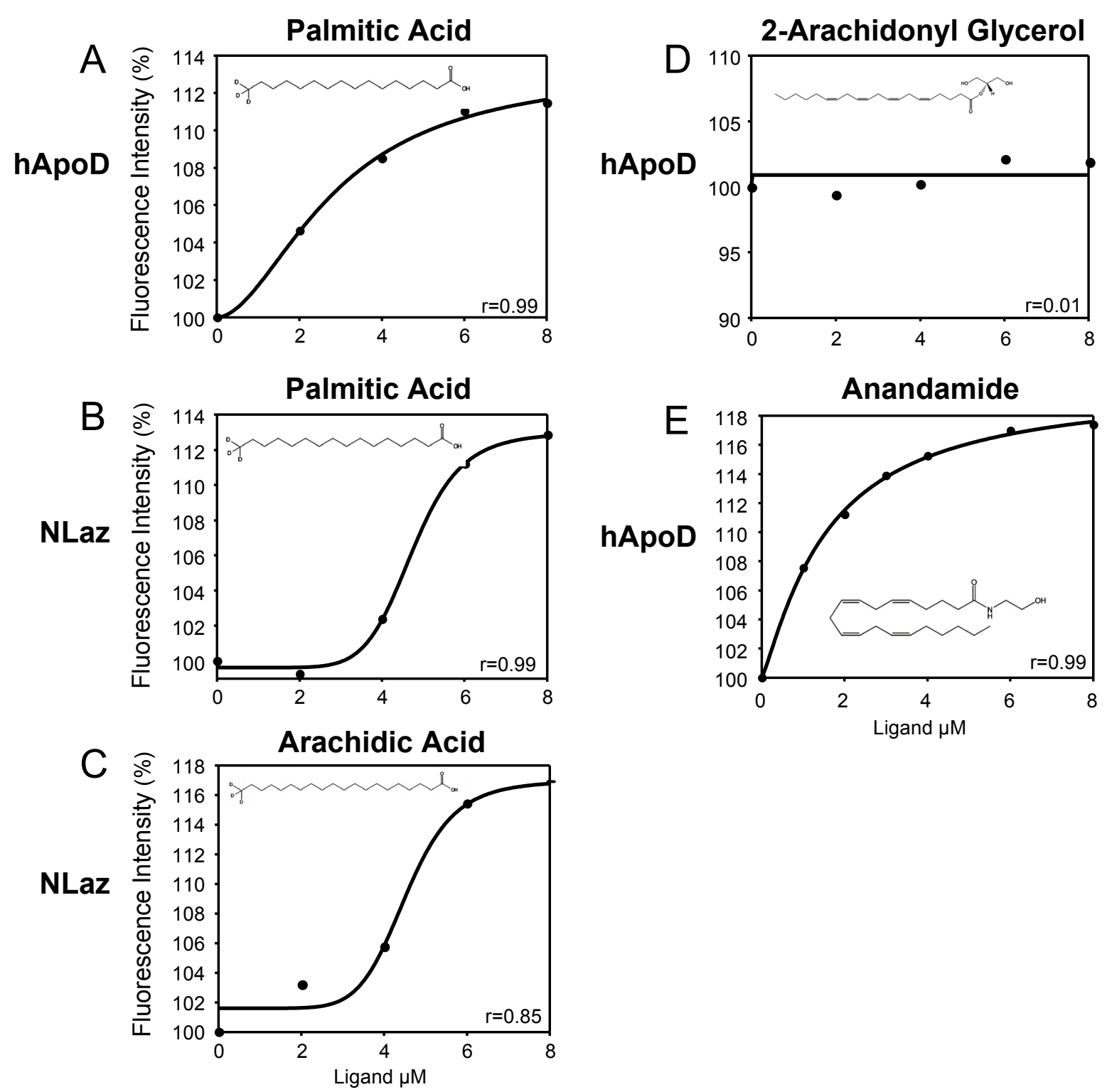
Fig. 5
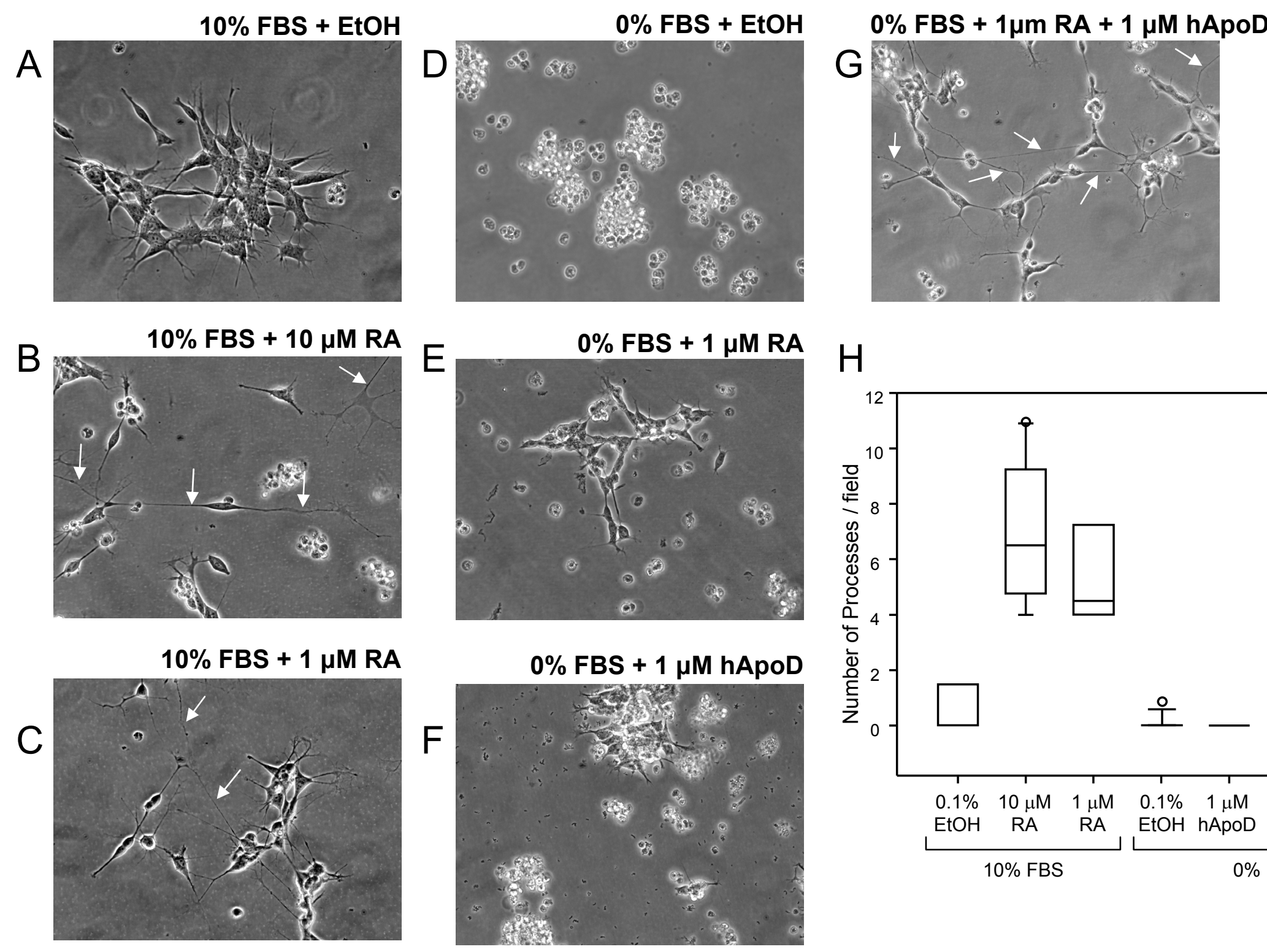

$\mathrm{H}$

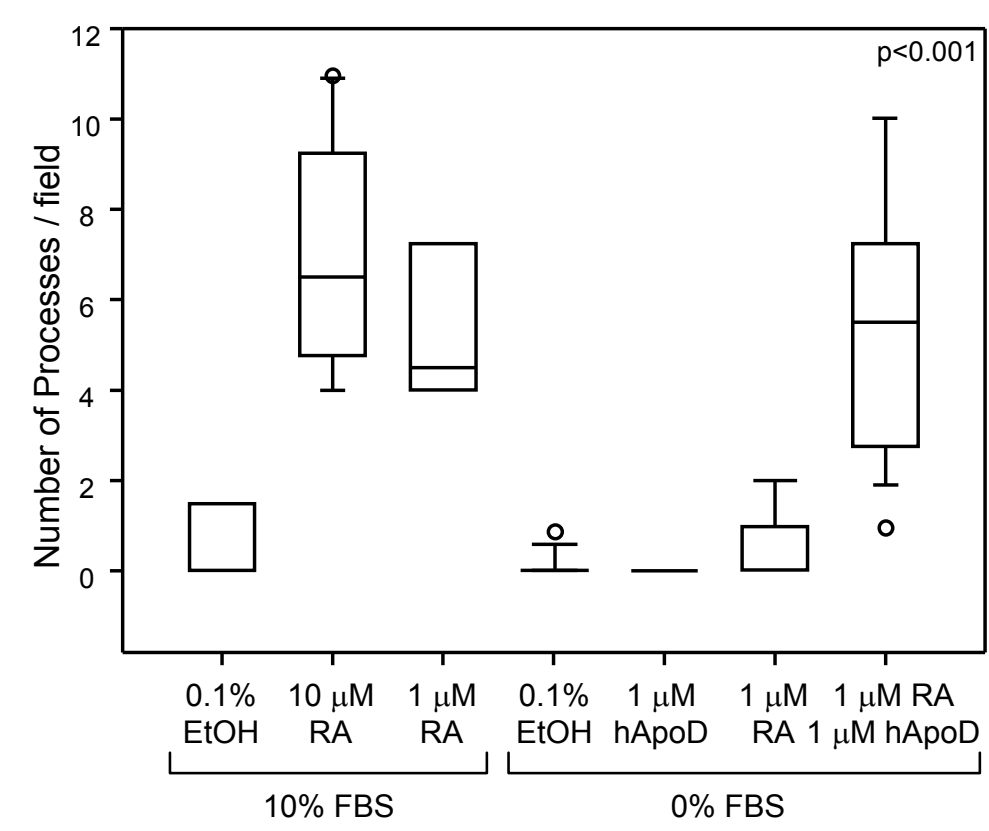


Fig. 6

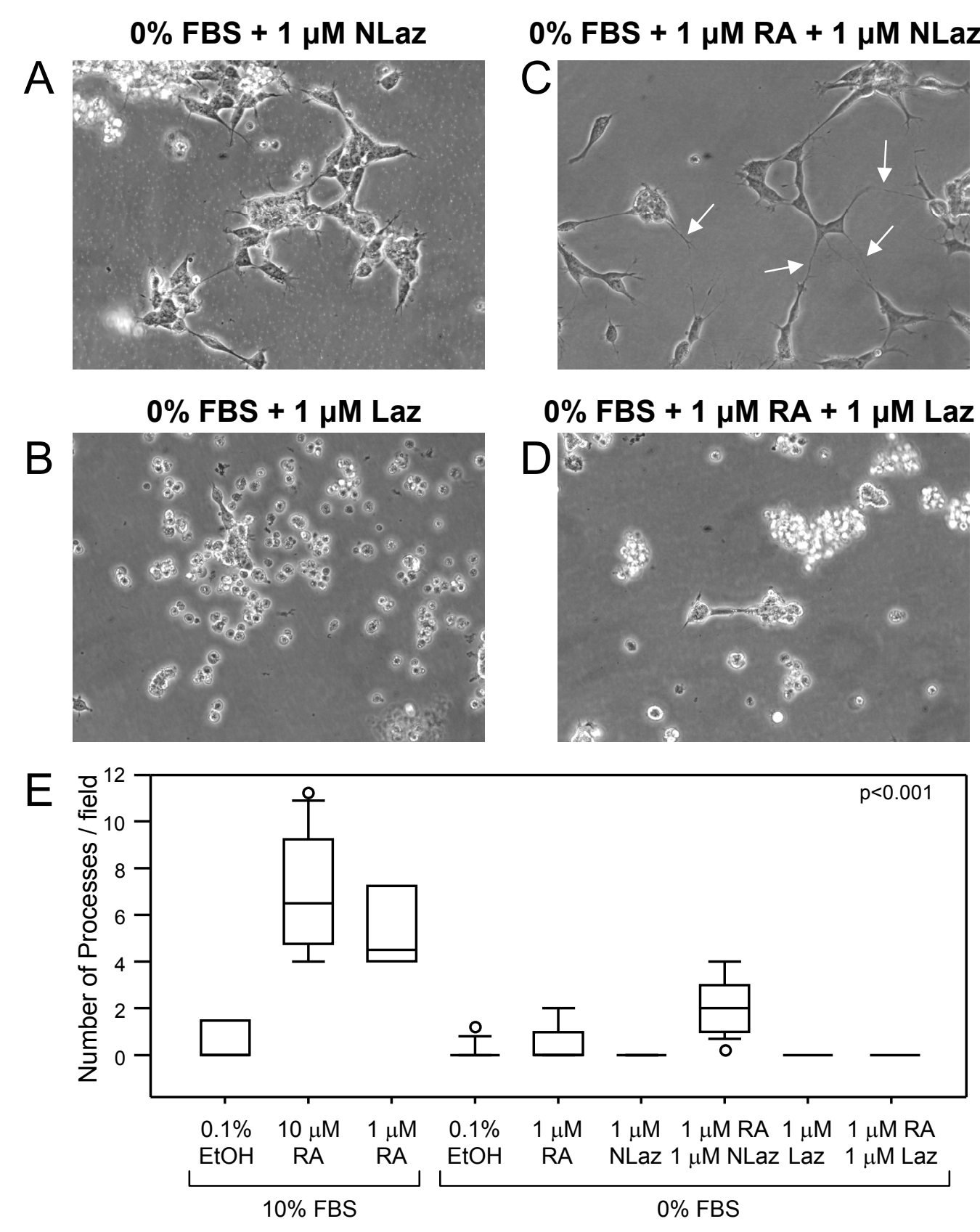


Fig. 7
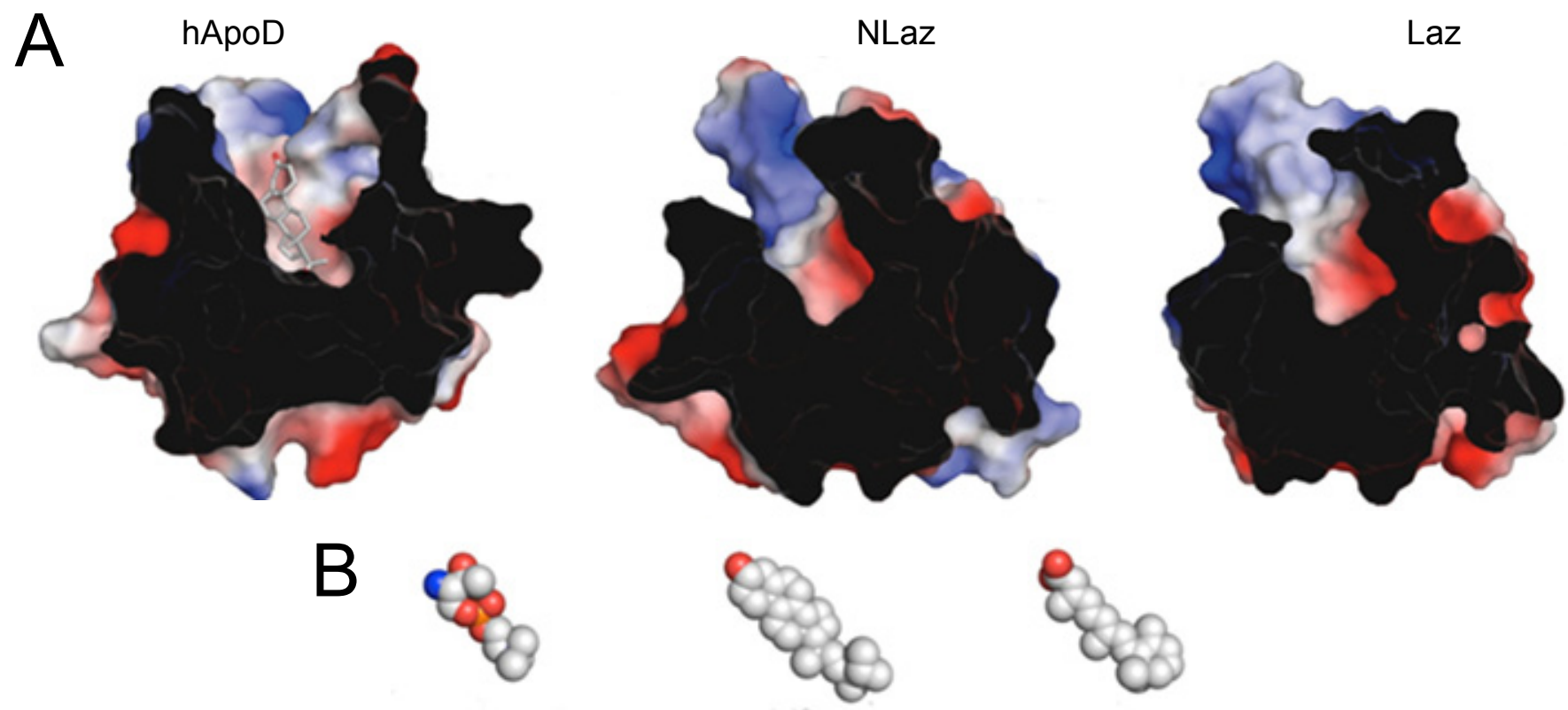

SM head group

Ergosterol

RA

C Hsap.APOD -QAFHLGKCPNPPVQENFDVNKYLGRWYEIEKIPTT-FENGR-CIQANYSLMENGKIKVLNQELRADGTV 67

SCR1 Dmel.NLaz AQVPFPGKCPDVKLLDTFDAEAYMGVWYEYAAYPFA-FEIGKKCIYANYSLIDNSTVSVVNAAINRFTGQ 69 $\longrightarrow$ SCR2 SCR3

Hsap. ApoD NQ-IEGEATPVNLTEP-AKLEVKFSWF--MPSAPYWILATDYENYALVYSCTCIIQ-LFHVDFAWILARNPNL 135 Dmel. NLaz PSNVTGQAKVLGPGQ---- LAVAFYPTQPLTKANYLVLGTDYESYAVVYSCTSVTP-LANFKIVWILTRQREP 137 Same.Laz NT-TTGWAEFASELHT DGKLSVHFPN--SPSVGNYWILSTDYDNYSIVWSCVKRPDSAASTEISWILLRSRNS 138 $\overbrace{2}^{2}$

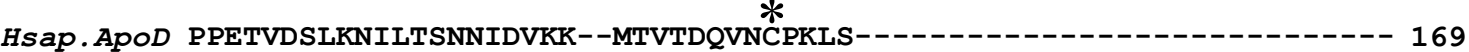
Dmel.NLaz SAEAVDAARKILEDNDVSQAF--LIDTVQKNCPRLDGNGTGLAGEDGLDVDDFVSTTVPNAIEKA 200

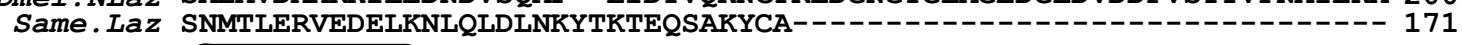
0 
Fig. S1
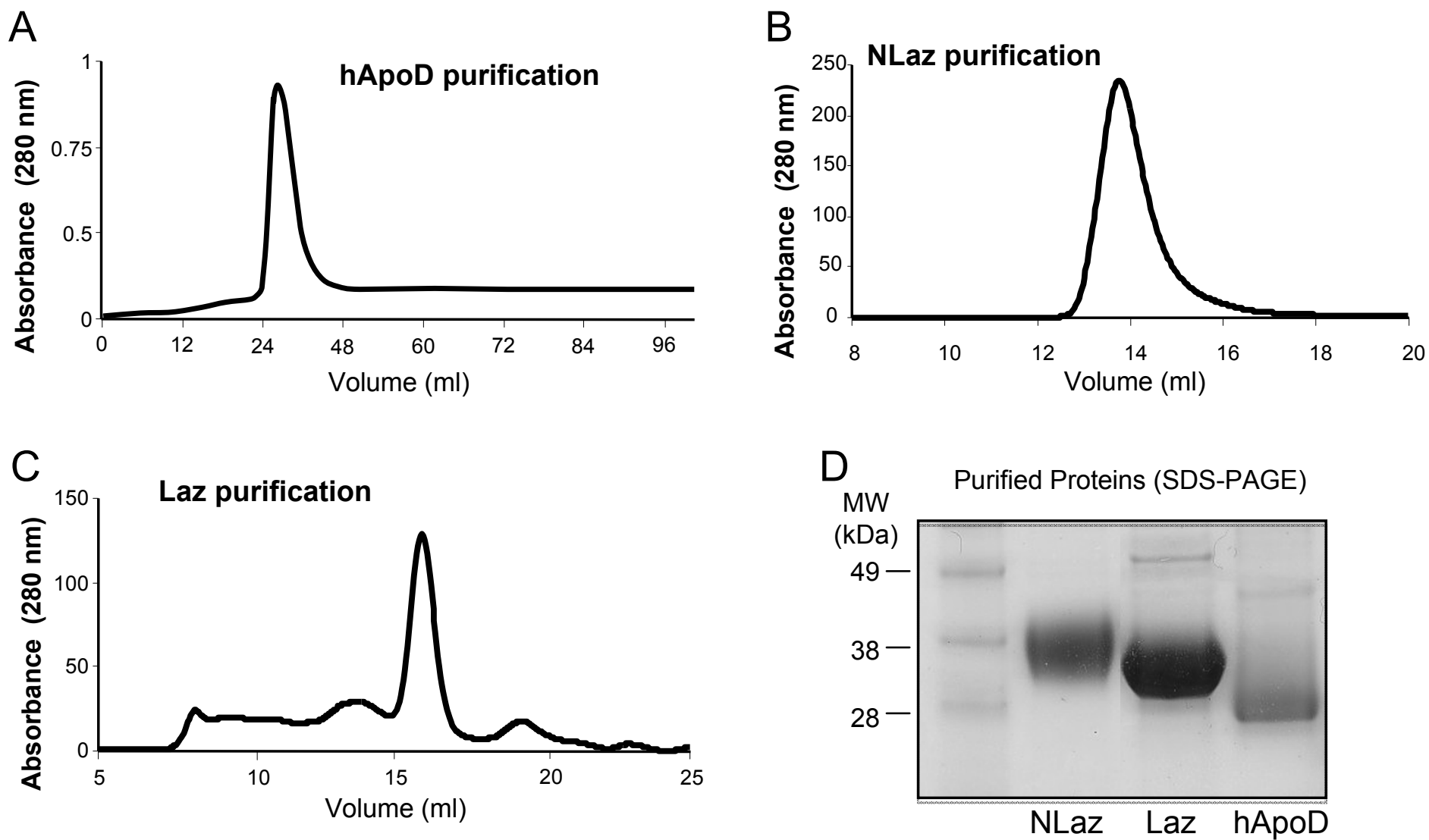

\section{Fig.S1.}

Expression and purification of hApoD from human breast cystic fluid, and of NLaz and Laz from S2 cells. Size-exclusion chromatography elution profile of hApoD (P60) (A), NLaz (analytical grade-200 column) (B), and Laz (analytical grade-200 column) (C). (D) SDS-PAGE analysis of the purified Lipocalins. 
Fig. S2
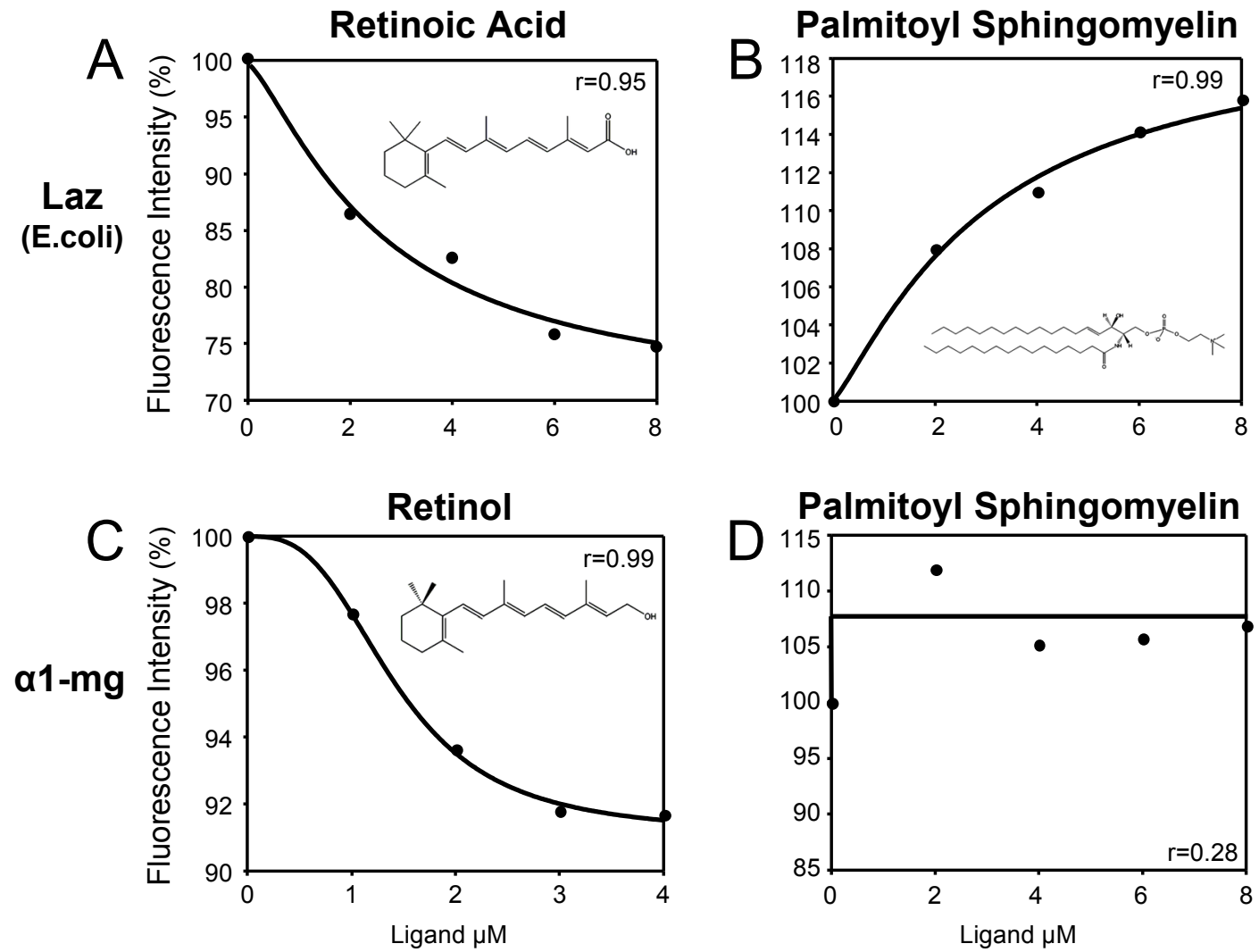

\section{Fig S2.}

Additional intrinsic fluorescence quenching studies. Recombinant Laz purified from $E$. coli reproduces the binding pattern of Laz purified from $S 2$ cells. Laz binds retinoic acid (A) and palmitoyl-sphingomyelin (B). A different Lipocalin (a1-mg) also binds retinol (C), but does not bind palmitoyl-sphingomyelin (D). 
Fig. S3
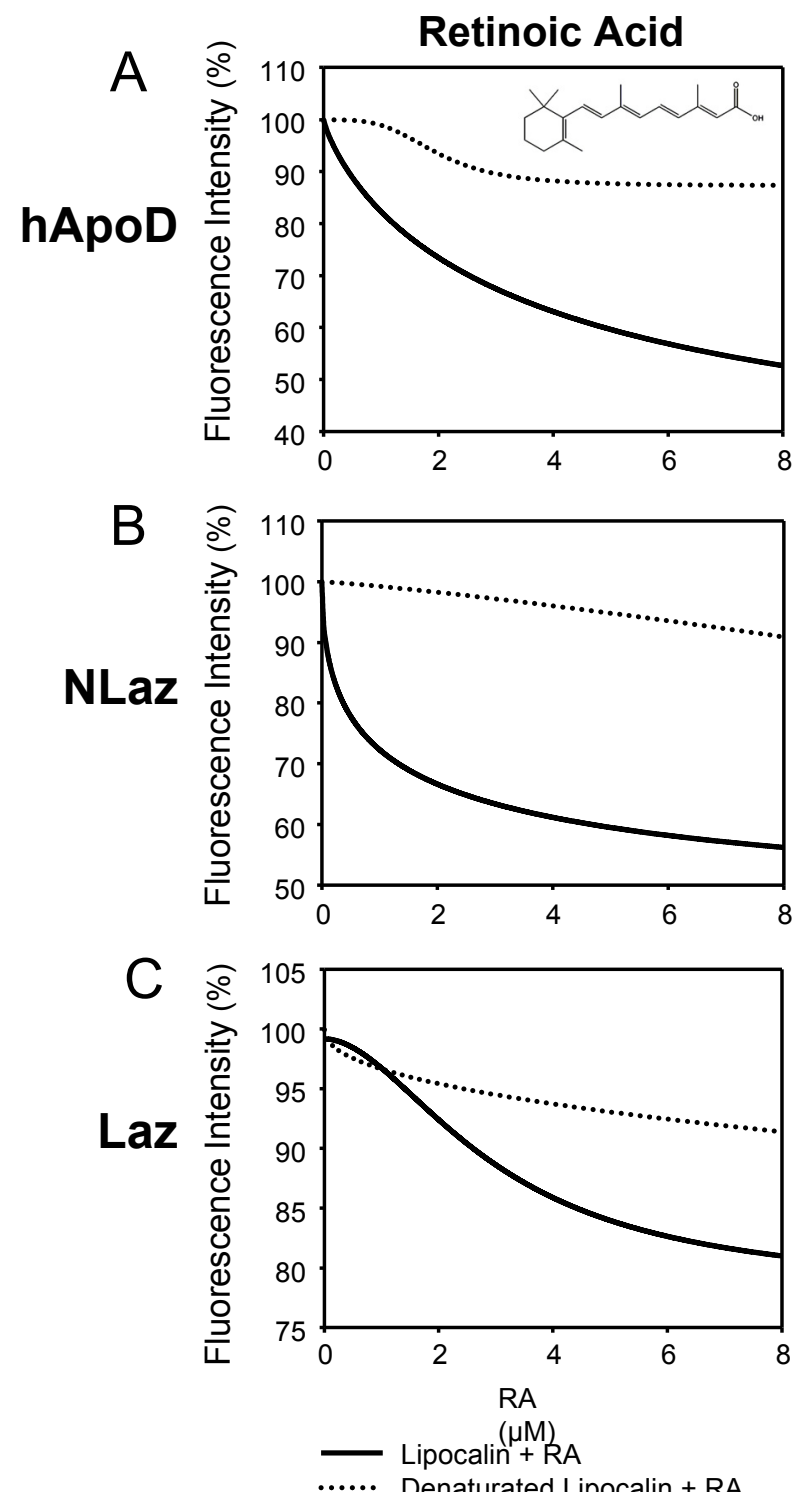

Fig.S3.

Lack of retinoic acid binding to $h A p o D$ (A), NLaz (B) and Laz (C) under denaturing conditions. Binding assay was performed either in the standard buffer or after $21 \mathrm{~h}$ incubation with $5 \mathrm{M}$ guanidine hydrochloride. Lipocalins-RA interaction depends on the protein tertiary structure. 
Fig. S4
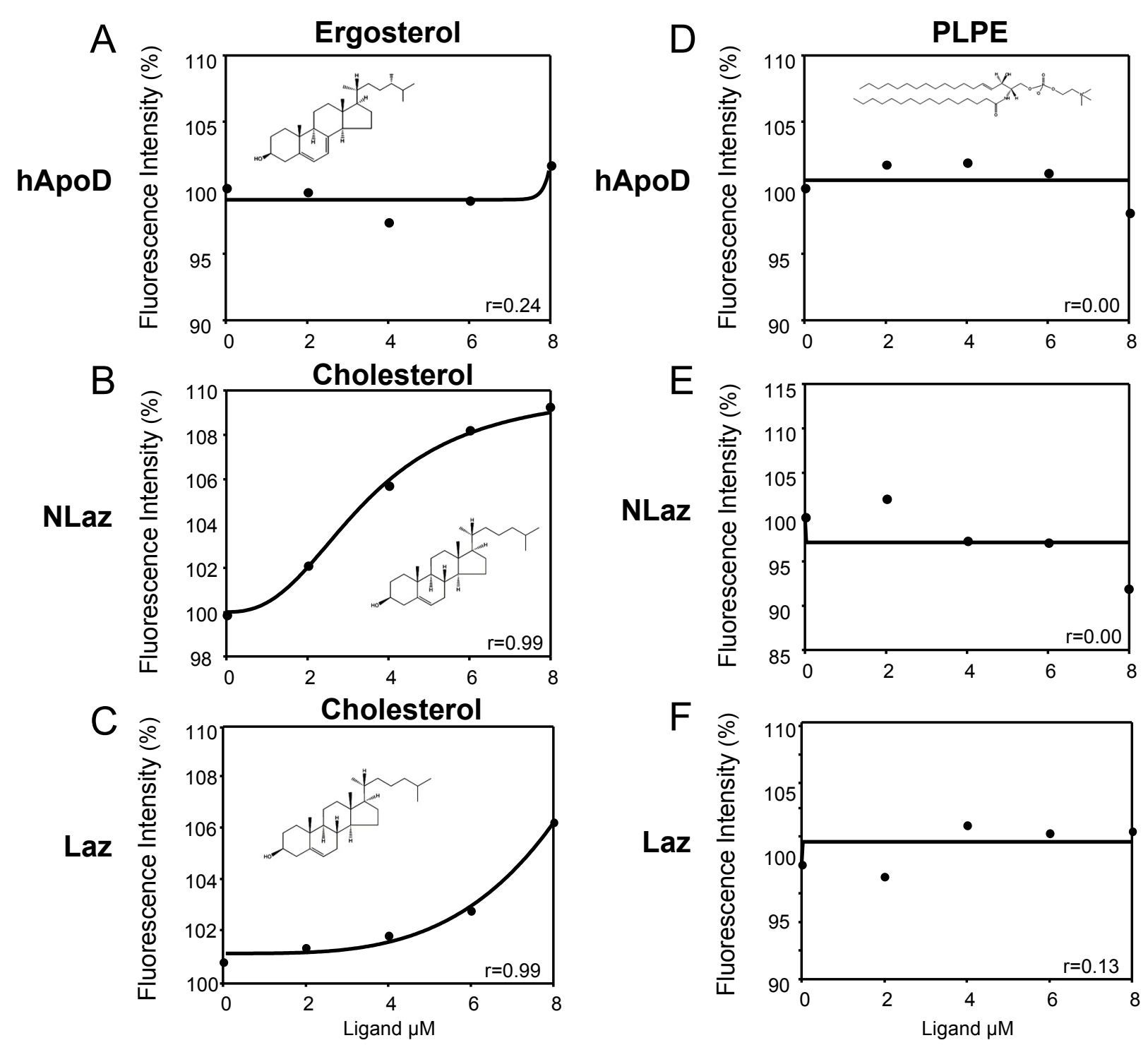

Fig S4.

Additional intrinsic fluorescence quenching studies. hApoD cannot bind ergosterol (A). NLaz (B), but not Laz (C) binds cholesterol. Neither hApoD (D), NLaz (E), nor Laz (F) are able to bind the phospholipid PLPE. 
Fig. S5
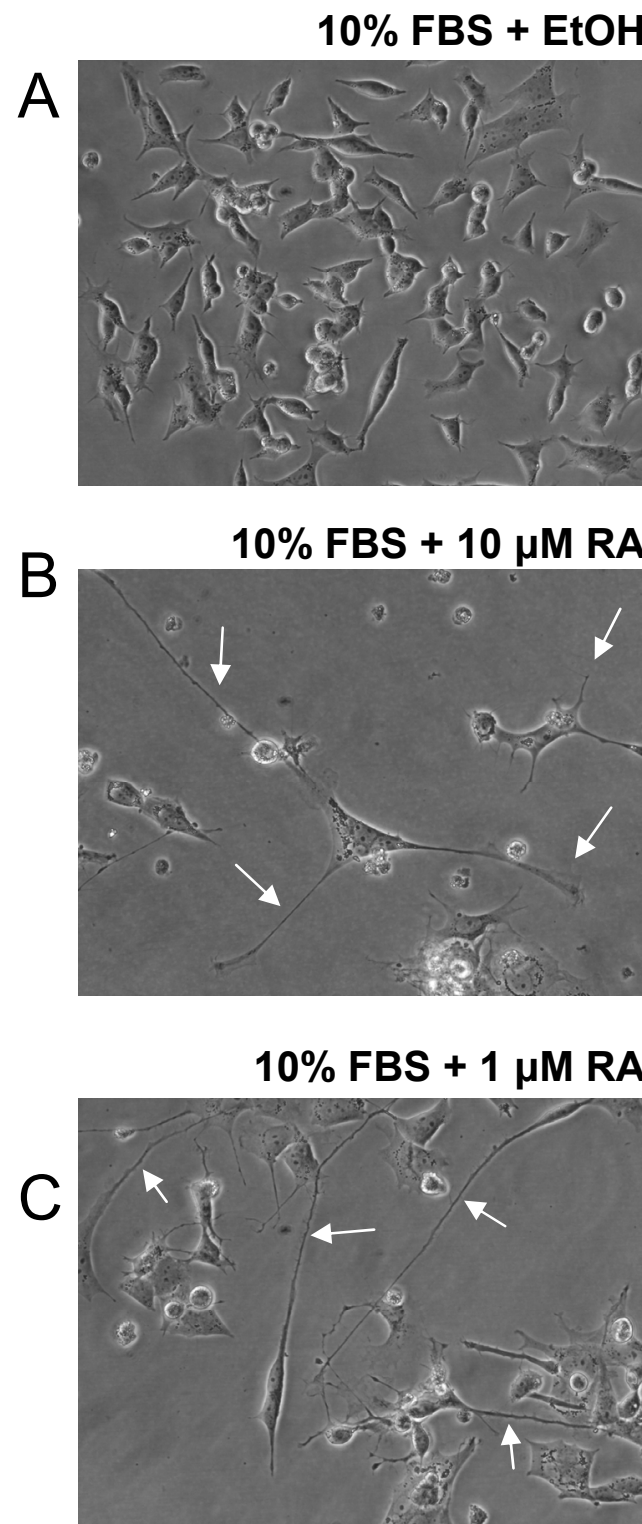

0\% FBS + EtOH
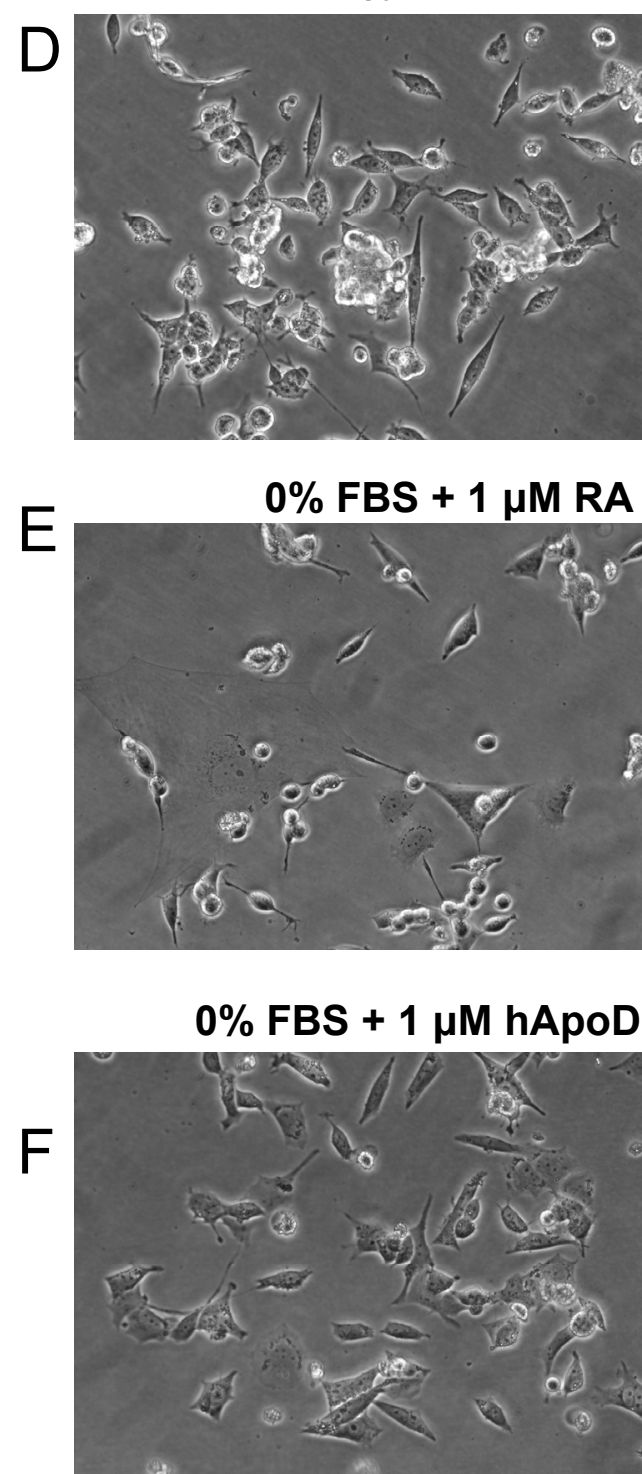

$0 \%$ FBS $+1 \mu \mathrm{m} \mathrm{RA}+1 \mu \mathrm{M}$ hApoD

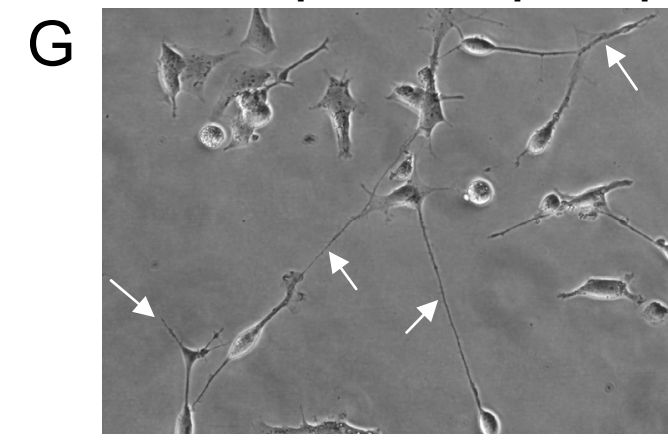

$\mathrm{H}$

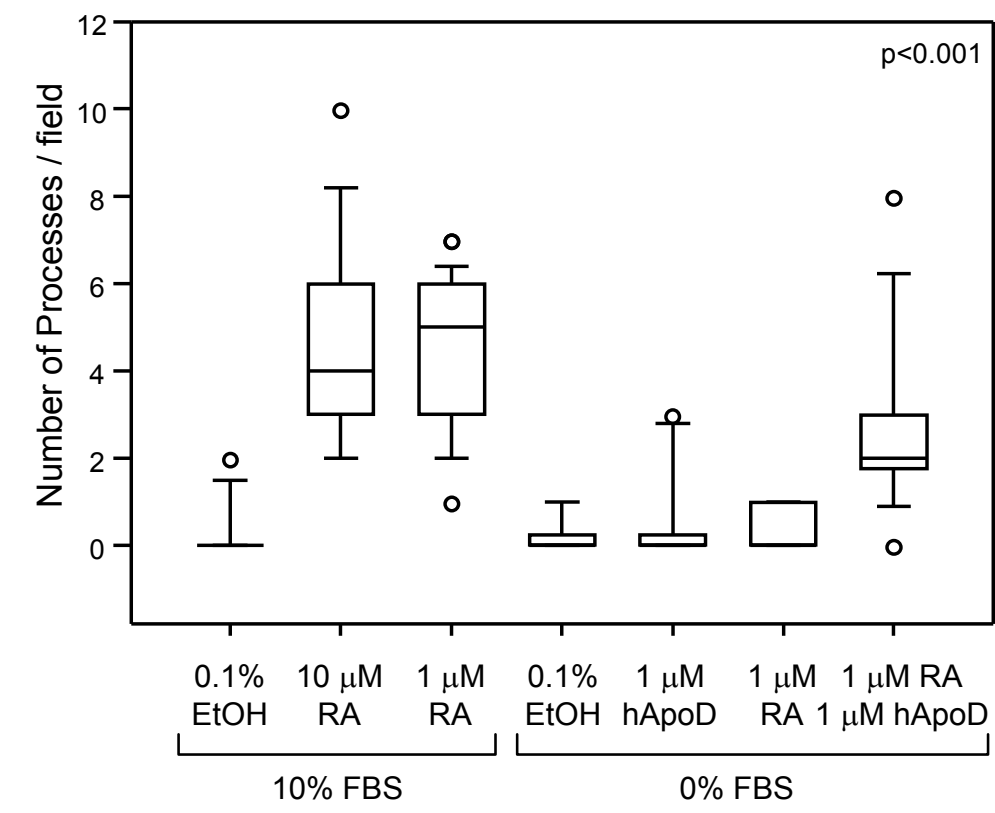

Page 1 of 2 


\section{Fig S5.}

Confirmation of the physiological role of RA and hApoD in M17 cells, a different human neuronal cell line. Morphology of M17 cells growing in complete medium with ethanol carrier (A), and after treatment with $10 \mu \mathrm{M}$ RA (B) or $1 \mu \mathrm{M}$ RA (C) in complete medium. Cells in Serum-free medium with ethanol carrier (D); Cell differentiation after 4-6 days of treatmet with $1 \mu \mathrm{M} R A(E), 1 \mu \mathrm{M} h \mathrm{hpoD}(\mathrm{F})$, or $1 \mu \mathrm{M}$ RA plus $1 \mathrm{mM}$ hApoD (G). (H) Quantification of the number of neurite processes observed per field normalized by the number of cells per field in each condition. Bars represent the quantification of 10-25 fields per condition from 3 independent experiments. Kruskal-Wallis one-way ANOVA on Ranks $(p<0.001)$ followed by Dunn's multiple comparison post-hoc test. In M17 cells, hApoD is also able to substitute for serum and to allow maturation and neurite growth. 
Fig. S6
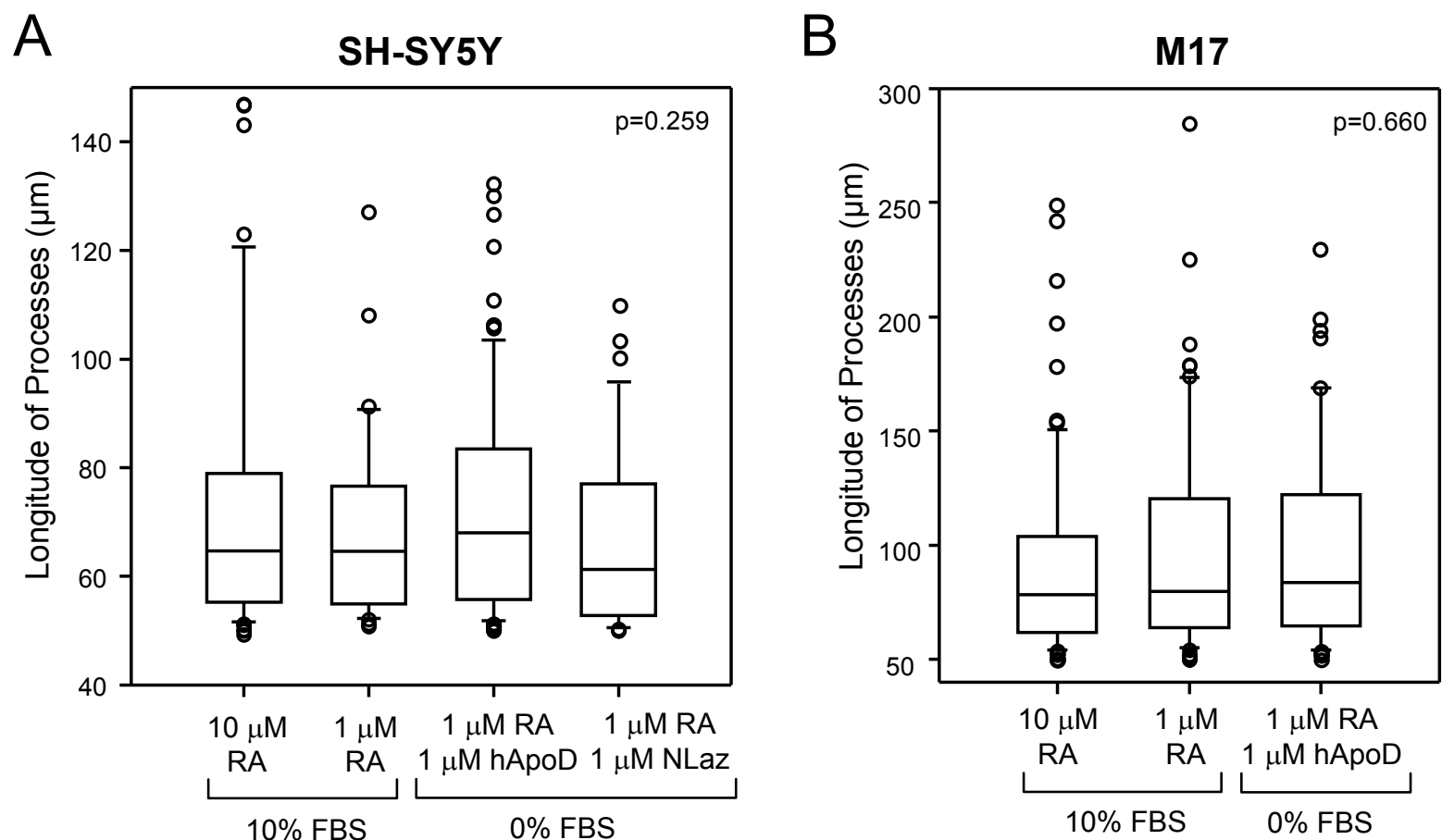

\section{Fig S6.}

Quantification of neurite length upon retinoic acid induced differentiation of SH-SY5Y (A) or M17 (B) neuronal cell lines. No changes are observed in the length of neurites between treatments. Kruskal-Wallis one-way ANOVA on Ranks ( $p>0.05)$. 
Table 1. Comparison of apparent $K_{D}$ values for the binding of various ligands to Lipocalins (average value \pm S.D.).

\begin{tabular}{|c|c|c|c|}
\hline Protein & Ligand & $K_{D}(\mu M)$ & $\mathbf{n}$ \\
\hline \multirow{10}{*}{ hApoD } & All-trans-Retinoic Acid & 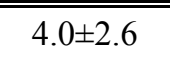 & 8 \\
\hline & Retinol & $0.2 \pm 0.1$ & 7 \\
\hline & Cholesterol & $\mathrm{n} / \mathrm{d}$ & 3 \\
\hline & Ergosterol & $\mathrm{n} / \mathrm{d}$ & 4 \\
\hline & Palmitoyl Sphingomyelin & $1.3 \pm 0.5$ & 6 \\
\hline & Palmitic Acid & $3.3 \pm 0.6$ & 8 \\
\hline & PLPE & $\mathrm{n} / \mathrm{d}$ & 3 \\
\hline & $\beta$-Estradiol & $\mathrm{n} / \mathrm{d}$ & 7 \\
\hline & 2-Arachidonyl-Glycerol & $\mathrm{n} / \mathrm{d}$ & 3 \\
\hline & Anandamide & $1.6 \pm 1.3$ & 10 \\
\hline \multirow{12}{*}{ NLaz } & All-trans-Retinoic Acid & $1.4 \pm 1.6$ & 9 \\
\hline & Retinol & $0.9 \pm 1.2$ & 10 \\
\hline & Cholesterol & $3.5 \pm 0.5$ & 4 \\
\hline & Ergosterol & $2.7 \pm 0.7$ & 6 \\
\hline & Palmitoyl Sphingomyelin & $4.6 \pm 0.2$ & 8 \\
\hline & Palmitic Acid & $4.7 \pm 0.2$ & 6 \\
\hline & Arachidic Acid & $4.5 \pm 0.7$ & 3 \\
\hline & PLPE & $\mathrm{n} / \mathrm{d}$ & 8 \\
\hline & 7(Z)-Tricosene & $4.5 \pm 0.6$ & 6 \\
\hline & 7(Z),(11)-Heptacosadiene & $\mathrm{n} / \mathrm{d}$ & 6 \\
\hline & 11-cis-Vaccenil-Acetate & $\mathrm{n} / \mathrm{d}$ & 5 \\
\hline & 20-Hydroxyecdysone & $\mathrm{n} / \mathrm{d}$ & 3 \\
\hline \multirow{6}{*}{ Lazarillo (S2) } & All-trans-Retinoic Acid & $3.0 \pm 1.1$ & 8 \\
\hline & Retinol & $2.1 \pm 0.6$ & 5 \\
\hline & Cholesterol & $\mathrm{n} / \mathrm{d}$ & 3 \\
\hline & Ergosterol & $2.0 \pm 0.1$ & 7 \\
\hline & Palmitoyl Sphingomyelin & $2.7 \pm 0.2$ & 7 \\
\hline & PLPE & $\mathrm{n} / \mathrm{d}$ & 5 \\
\hline \multirow{2}{*}{ Lazarillo (E.coli) } & All-trans-Retinoic Acid & $2.6 \pm 1.8$ & 3 \\
\hline & Palmitoyl Sphingomyelin & $3.0 \pm 2.6$ & 3 \\
\hline \multirow{2}{*}{ a1-microglobulin } & Retinol & $1.4 \pm 0.1$ & 4 \\
\hline & Palmitoyl Sphingomyelin & $\mathrm{n} / \mathrm{d}$ & 3 \\
\hline
\end{tabular}

$\mathrm{n}=$ number of independent ligand-titration experiments. 